Pacific

Journal of

Mathematics

BOTT FORMULA OF THE MASLOV-TYPE INDEX THEORY

YIMING LONG 


\title{
BOTT FORMULA OF THE MASLOV-TYPE INDEX THEORY
}

\author{
YIMING LONG
}

In this paper the integer valued $\omega$-index theory parameterized by all $\omega$ on the unit circle for paths in the symplectic group $\operatorname{Sp}(2 n)$ is established. Based on this index theory, the Bott formula of the Maslov-type index theory for iterated paths in $\operatorname{Sp}(2 n)$ is estalished, the mean index for periodic solutions of Hamiltonian systems is defined, and the increasing estimate of the iterated Maslov-type index in terms of the mean index is proved.

\section{Introduction and main results.}

For $n \in \mathbf{N}$, the set of natural numbers, as usual we define the symplectic group

$$
\operatorname{Sp}(2 n) \equiv \operatorname{Sp}(2 n, \mathbf{R})=\left\{M \in \mathcal{L}\left(\mathbf{R}^{2 n}\right) \mid M^{T} J M=J\right\},
$$

where $J=\left(\begin{array}{cc}0 & -I_{n} \\ I_{n} & 0\end{array}\right), I_{n}$ is the identity matrix on $\mathbf{R}^{n}$, and $\mathcal{L}\left(\mathbf{R}^{2 n}\right)$ is the space of $2 n \times 2 n$ real matrices. When there is no confusion, we shall omit the subindex of the identity matrices. The metric on $\operatorname{Sp}(2 n)$ is inherited through $\mathcal{L}\left(\mathbf{R}^{2 n}\right)$ from that of $\mathbf{R}^{4 n^{2}}$. For $\tau>0$, suppose $H \in C^{2}\left(S_{\tau} \times \mathbf{R}^{2 n}, \mathbf{R}\right)$, where $S_{\tau} \equiv \mathbf{R} /(\tau \mathbf{Z})$. Let $x$ be a $\tau$-periodic solution of the nonlinear Hamiltonian system

$$
\dot{x}(t)=J H^{\prime}(t, x(t)), \quad x \in \mathbf{R}^{2 n} .
$$

Then the fundamental solution of the linearized Hamiltonian system

$$
\dot{y}=J B(t) y, \quad y \in \mathbf{R}^{2 n},
$$

with $B(t)=H^{\prime \prime}(t, x(t))$, is a path $\gamma_{x} \in C^{1}([0,+\infty), \operatorname{Sp}(2 n))$ with $\gamma_{x}(0)=I$. In order to study the property of periodic solutions of (1.1), C. Conley and E. Zehnder defined their index theory for nondegenerate paths in $\operatorname{Sp}(2 n)$ starting from the identity matrix in their celebrated work $[\mathbf{C Z}]$ for the case of $n \geq 2$. This index theory was extended by E. Zehnder and the author in $[\mathbf{L Z}]$ to nondegenerate paths in $\operatorname{Sp}(2)$, by the author in [Lo1] and C. Viterbo in $[\mathbf{V i 2}]$ independently via different methods to degenerate paths which are fundamental solutions of linear Hamiltonian systems (1.2) for any $B \in C\left(S_{\tau}, \mathcal{L}_{s}\left(\mathbf{R}^{2 n}\right)\right)$, where $\mathcal{L}_{s}\left(\mathbf{R}^{2 n}\right)$ is the set of all real symmetric 
$2 n \times 2 n$ matrices, and recently by the author in [Lo8] to any degenerate paths in $\operatorname{Sp}(2 n)$ using ideas of [Lo1] and [Lo5] together with an axiom characterization of this index theory. In this paper we call it the Maslovtype index theory. Denote by

$$
\mathcal{P}_{\tau}(2 n)=\{\gamma \in C([0, \tau], \operatorname{Sp}(2 n)) \mid \gamma(0)=I\} .
$$

This index theory assigns a pair of integers $\left(i_{\tau}(\gamma), \nu_{\tau}(\gamma)\right) \in \mathbf{Z} \times\{0, \ldots, 2 n\}$ to each $\gamma \in \mathcal{P}_{\tau}(2 n)$. For any $\gamma \in \mathcal{P}_{\tau}(2 n)$, define the iteration path $\tilde{\gamma} \in$ $C([0,+\infty), \operatorname{Sp}(2 n))$ of $\gamma$ by

$$
\tilde{\gamma}(t)=\gamma(t-j \tau) \gamma(\tau)^{j}, \quad \text { for } j \tau \leq t \leq(j+1) \tau \text { and } j \in\{0\} \cup \mathbf{N} .
$$

Let $\gamma^{k}=\left.\tilde{\gamma}\right|_{[0, k \tau]}$. Then we can associate to $\gamma$ through $\tilde{\gamma}$ a sequence of Maslov-type indices $\left\{\left(i_{k \tau}(\tilde{\gamma}), \nu_{k \tau}(\tilde{\gamma})\right)\right\}_{k \in \mathbf{N}}$. When $\gamma:[0,+\infty) \rightarrow \operatorname{Sp}(2 n)$ is the fundamental soluton of (1.2) with $B \in C\left(S_{\tau}, \mathcal{L}_{s}\left(\mathbf{R}^{2 n}\right)\right)$, there holds $\left(\left.\gamma\right|_{[0, \tau]}\right)^{\sim}=\gamma$. Thus for $B(t)=H^{\prime \prime}(t, x(t))$ this index sequence with $\gamma=\gamma_{x}$ reflects important properties of the $\tau$-periodic solution $x$ of (1.1).

In order to gain more information on the iterated Maslov-type indices, in Section 2 of this paper, the Maslov-type index theory is generalized to the $\omega$-index theory for all $\omega \in \mathbf{U}$, where $\mathbf{U}$ is the unit circle in the complex plane C. This $\omega$-index theory assigns a pair of integers $\left(i_{\tau, \omega}(\gamma), \nu_{\tau, \omega}(\gamma)\right) \in$ $\mathbf{Z} \times\{0, \ldots, 2 n\}$ to each $\gamma \in \mathcal{P}_{\tau}(2 n)$. When $\omega=1$, the $\omega$-index theory coincides with the above mentioned Maslov-type index theory. The following are our main results on the $\omega$-index theory in this paper.

Definition 1.1. For any $M \in \operatorname{Sp}(2 n)$, define the homotopy set of $M$ in $\operatorname{Sp}(2 n)$ by

$$
\begin{aligned}
\Omega(M)= & \{N \in \operatorname{Sp}(2 n) \mid \sigma(N) \cap \mathbf{U}=\sigma(M) \cap \mathbf{U}, \text { and } \\
& \left.\operatorname{dim}_{\mathbf{C}} \operatorname{ker}_{\mathbf{C}}(N-\lambda I)=\operatorname{dim}_{\mathbf{C}} \operatorname{ker}_{\mathbf{C}}(M-\lambda I), \forall \lambda \in \sigma(M) \cap \mathbf{U}\right\} .
\end{aligned}
$$

We denote by $\Omega^{0}(M)$ the path connected component of $\Omega(M)$ which contains $M$, and call it the homotopy component of $M$ in $\operatorname{Sp}(2 n)$. For $\omega \in \mathbf{U}$, define

$$
\Omega_{\omega}(M)=\left\{N \in \operatorname{Sp}(2 n) \mid \operatorname{dim}_{\mathbf{C}} \operatorname{ker}_{\mathbf{C}}(N-\omega I)=\operatorname{dim}_{\mathbf{C}} \operatorname{ker}_{\mathbf{C}}(M-\omega I)\right\} .
$$

We denote by $\Omega_{\omega}^{0}(M)$ the path connected component of $\Omega_{\omega}(M)$ which contains $M$, and call it the $\omega$-homotopy component of $M$ in $\operatorname{Sp}(2 n)$.

For any $M \in \operatorname{Sp}(2 n)$, define $[M]=\left\{N \in \operatorname{Sp}(2 n) \mid N=P^{-1} M P\right.$ for some $P \in \operatorname{Sp}(2 n)\}$. Then $[M] \subset \Omega^{0}(M) \subset \Omega_{\omega}^{0}(M)$ for all $\omega \in \mathbf{U}$.

Theorem 1.2. For any $\tau>0, \gamma \in \mathcal{P}_{\tau}(2 n)$, and $\omega \in \mathbf{U}$, the $\omega$-index pair $\left(i_{\tau, \omega}(\gamma), \nu_{\tau, \omega}(\gamma)\right)$ of $\gamma$ is completely determined by the homotopy class of $\gamma$ in $\mathcal{P}_{\tau}(2 n)$ in the sense of Definition 2.2 below. More specially, $i_{\tau, \omega}(\gamma)$ is completely determined by the $\omega$-homotopy component $\Omega_{\omega}^{0}(\gamma(\tau))$ of $\gamma(\tau)$ in 
$\operatorname{Sp}(2 n)$ up to an additive constant, and $\nu_{\tau, \omega}(\gamma)$ is completely determined by $\Omega_{\omega}^{0}(\gamma(\tau))$.

The idea of our $\omega$-index theory is closely related to those of $[\mathbf{C Z}],[\mathbf{L Z}]$, $[\mathbf{L o 1}, \mathbf{L o 2}, \mathbf{L o 5}, \mathbf{L o 8}]$, and [DL], but is very different from those of [Bo], $[\mathbf{E d}],[\mathbf{C D}],[\mathbf{E k}]$, and [Vi1]. Our method is rather elementary and intuitive. The establishment of the $\omega$-index theory and the proof of Theorem 1.2 are based on the understanding of the topological structures of the singular hypersurface $\operatorname{Sp}(2 n)_{\omega}^{0}$, which contains all symplectic matrices possessing the eigenvalue $\omega$, and its complement $\operatorname{Sp}(2 n)_{\omega}^{*}$ in $\operatorname{Sp}(2 n)$ obtained in the papers $[\mathbf{C Z}],[\mathbf{L Z}],[\mathbf{L o 1}]-[\mathbf{L o 6}]$, and $[\mathbf{L D}]$. Fix $\omega \in \mathbf{U}$. For any $\gamma \in \mathcal{P}_{\tau}(2 n)$ we define $\nu_{\tau, \omega}(\gamma)$ to be $\operatorname{dim}_{\mathbf{C}} \operatorname{ker}_{\mathbf{C}}(\gamma(\tau)-\omega I)$. Since $\operatorname{Sp}(2 n)$ is homeomorphic to a product of $\mathbf{U}$ and a simply connected space, any path $\gamma \in \mathcal{P}_{\tau}(2 n)$ can be viewed as rotating around the unit circle part in $\operatorname{Sp}(2 n)$. Because $\operatorname{Sp}(2 n)_{\omega}^{*}$ possesses precisely two path connected components and $\operatorname{Sp}(2 n)_{\omega}^{*}$ is simply connected in $\operatorname{Sp}(2 n)$, we can give a way to count this rotation suitably for every non-degenerate path $\gamma$, i.e. $\omega \notin \sigma(\gamma(\tau))$, which defines the $\omega$ index $i_{\tau, \omega}(\gamma) \in \mathbf{Z}$. For any degenerate path $\gamma \in \mathcal{P}_{\tau}(2 n)$, i.e. $\omega \in \sigma(\gamma(\tau))$, using the topology of $\operatorname{Sp}(2 n)$ near $\operatorname{Sp}(2 n)_{\omega}^{0}$, we construct topologically two families of non-degenerate paths $\gamma_{-s}$ and $\gamma_{s}$ near $\gamma$ for $s \in(0,1]$ satisfying $i_{\tau, \omega}\left(\gamma_{s}\right)-i_{\tau, \omega}\left(\gamma_{-s}\right)=\nu_{\tau, \omega}(\gamma)$. We also prove analytically in Section 4 that $\gamma_{-s}$ and $\gamma_{s}$ takes the minimum and maximum values of $i_{\tau, \omega}$ among all nondegenerate path in $\mathcal{P}_{\tau}(2 n)$ near $\gamma$. Then we define this minimum to be $i_{\tau, \omega}(\gamma)$. In Theorem 2.11 below, we give an axiom characterization of the $\omega$-index theory as an integer valued map defined on $\cup_{n \geq 1} \mathcal{P}_{\tau}(2 n)$ via the homotopy invariance, symplectic additivity, and the values of it on $\mathcal{P}_{\tau}(2)$.

In Section 4 we introduce the concept of splitting numbers:

Theorem 1.3. For any $M \in \operatorname{Sp}(2 n)$ and $\omega \in \mathbf{U}$, choose $\tau>0$ and $\gamma \in \mathcal{P}_{\tau}(2 n)$ with $\gamma(\tau)=M$, and define

$$
S_{M}^{ \pm}(\omega)=\lim _{\epsilon \rightarrow 0^{+}} i_{\tau, \exp ( \pm \epsilon \sqrt{-1}) \omega}(\gamma)-i_{\tau, \omega}(\gamma)
$$

Then these two integers are independent of the choice of the path $\gamma$. They are called the splitting numbers of $M$ at $\omega$.

We also prove that the splitting numbers coincide with the ultimate type of $\omega \in \mathbf{U}$ for $M$, a new concept introduced in the following Definition 4.8. Specially we prove that splitting numbers of $M$ at $\omega$ are constants on $\Omega^{0}(M)$, and give a complete characterization of splitting numbers.

Based on the $\omega$-index theory and the idea of R. Bott in [Bo], in Section 5 we are able to establish the following Bott-type formulae of the Maslov-type index theory for iterations of any path in $\mathcal{P}_{\tau}(2 n)$.

Theorem 1.4. For any $\tau>0, \gamma \in \mathcal{P}_{\tau}(2 n)$, and $k \in \mathbf{N}$, there hold 


$$
\begin{aligned}
& i_{k \tau}(\tilde{\gamma})=i_{k \tau, 1}(\tilde{\gamma})=\sum_{\omega^{k}=1} i_{\tau, \omega}(\gamma), \\
& \nu_{k \tau}(\tilde{\gamma})=\nu_{k \tau, 1}(\tilde{\gamma})=\sum_{\omega^{k}=1} \nu_{\tau, \omega}(\gamma) .
\end{aligned}
$$

As a direct consequence of Theorem 1.4, the mean index per $\tau$ for any path $\gamma \in \mathcal{P}_{\tau}(2 n)$ can be defined and an estimate on $i_{k \tau}$ is obtained.

Theorem 1.5. For any $\tau>0$ and $\gamma \in \mathcal{P}_{\tau}(2 n)$ there hold

$$
\begin{aligned}
& \hat{i}_{\tau}(\gamma) \equiv \lim _{k \rightarrow+\infty} \frac{i_{k \tau}(\tilde{\gamma})}{k}=\frac{1}{2 \pi} \int_{\mathbf{U}} i_{\tau, \omega}(\gamma) d \omega, \\
& \hat{\nu}_{\tau}(\gamma) \equiv \lim _{k \rightarrow+\infty} \frac{\nu_{k \tau}(\tilde{\gamma})}{k}=\frac{1}{2 \pi} \int_{\mathbf{U}} \nu_{\tau, \omega}(\gamma) d \omega=0 .
\end{aligned}
$$

Specially, $\hat{i}_{\tau}(\gamma)$ is always a finite real number, which is called the mean index per $\tau$ of $\gamma$.

Theorem 1.6. For any $\tau>0$ and $\gamma \in \mathcal{P}_{\tau}(2 n)$, there holds

$$
\left|k \hat{i}_{\tau}(\gamma)-i_{k \tau}(\tilde{\gamma})\right| \leq n, \quad \forall k \in \mathbf{N} .
$$

Then through the fundamental solution $\gamma_{x}$ of (1.2) with $B(t)=H^{\prime \prime}(t, x(t))$ the mean index per period $\tau$ of a $\tau$-periodic solution $x$ of the nonlinear system (1.1) can be defined by $\hat{i}_{\tau}(x)=\hat{i}_{\tau}\left(\gamma_{x}\right)$. When $\tau$ is the minimal period of $x$, we denote by $\hat{i}(x)=\hat{i}_{\tau}(x)$. This yields a new invariant to each periodic solution of the system (1.1).

Since Bott's celebrated work [Bo] in 1956, iteration properties of various Morse and Maslov type index theories have been studied by $[\mathbf{E d}],[\mathbf{C D}]$, $[\mathbf{K l}],[\mathbf{E k}],[\mathbf{V i 1}],[\mathbf{D L}],[\mathbf{L o 5}]$, and others. But upto the author's knowledge, the Bott-type formula were established only by H. Edwards for the Sturm form with finite Morse indices (cf. [Ed]), by I. Ekeland (cf. [Ek]) for convex Hamiltonian systems, and by C. Viterbo (cf. [Vi1]) for star-shaped Hamiltonian systems under certain nondegeneracy conditions. In Section 6 , we prove that our $\omega$-index theory generalizes the Bott functions $\Lambda(\omega)$ and $N(\omega)$ defined in [Bo] and Ekeland's index functions defined in [Ek]. Therefore from Theorem 1.4, we recover their formulae for iterations. Our results also generalizes those in [Vi1]. Results obtained in the current paper have been applied to the study of the existence, multiplicity, and stability of periodic solutions of nonconvex Hamiltonian systems in our other papers.

In Section 7, for reders convenience, we briefly introduce results obtained in $[\mathbf{L D}]$ on the basic normal forms of symplectic matrices, in [Lo6] on the topological structures of the hypersurface $\operatorname{Sp}(2 n)_{\omega}^{0}$ and its complement in $\operatorname{Sp}(2 n)$, and study properties of homotopy components of symplectic matrices, which are fundamental for our discussion in this paper. 


\section{The $\omega$-index theory.}

In this section, we define the $\omega$-index theory as a generalization of the Maslov-type index theory, and study its basic properties. In the following we fix $n \in \mathbf{N}$ and use notations introduced in Section 7 .

For any $\tau>0$, we define the metric of $\mathcal{P}_{\tau}(2 n)$ to be the one induced from the usual metric of $C([0, \tau], \operatorname{Sp}(2 n))$. For $\omega \in \mathbf{U}$, we define two subsets of $\mathcal{P}_{\tau}(2 n)$ by

$$
\mathcal{P}_{\tau, \omega}^{*}(2 n)=\left\{\gamma \in \mathcal{P}_{\tau}(2 n) \mid \gamma(\tau) \in \operatorname{Sp}(2 n)_{\omega}^{*}\right\}, \quad \mathcal{P}_{\tau, \omega}^{0}(2 n)=\mathcal{P}_{\tau}(2 n) \backslash \mathcal{P}_{\tau, \omega}^{*}(2 n) .
$$

We define the following subfamilies of paths in $\mathcal{P}_{\tau}(2 n)$ which are fundamental solutions of Hamiltonian systems (1.2) with continuous symmetric and $\tau$ periodic coefficients.

$$
\begin{aligned}
\hat{\mathcal{P}}_{\tau}(2 n) & =\left\{\gamma \in \mathcal{P}_{\tau}(2 n) \cap C^{1}([0, \tau], \operatorname{Sp}(2 n)) \mid \dot{\gamma}(\tau)=\dot{\gamma}(0) \gamma(\tau)\right\}, \\
\hat{\mathcal{P}}_{\tau, \omega}^{*}(2 n) & =\hat{\mathcal{P}}_{\tau}(2 n) \cap \mathcal{P}_{\tau, \omega}^{*}(2 n), \quad \hat{\mathcal{P}}_{\tau, \omega}^{0}(2 n)=\hat{\mathcal{P}}_{\tau}(2 n) \backslash \hat{\mathcal{P}}_{\tau, \omega}^{*}(2 n) .
\end{aligned}
$$

Let the metric of $\hat{P}_{\tau}(2 n)$ be the one induced from the usual metric of $C^{1}([0, \tau], \operatorname{Sp}(2 n))$. Note that for any $\gamma \in \mathcal{P}_{\tau}(2 n)$, we can choose a $C^{1}$ path $\beta \in \mathcal{P}_{\tau}(2 n)$ possessing the same end points as $\gamma$ and as $C^{0}$-close to $\gamma$ as we want. Now composing $\beta$ with a suitable function $\rho \in C^{2}([0, \tau],[0,1])$ satisfying $\rho(0)=0, \dot{\rho}(0)=0, \rho(\tau)=1, \dot{\rho}(\tau)=0$, and $\dot{\rho}(t) \geq 0$ for all $0 \leq t \leq \tau$, without loss of generality, we can assume $\beta \in \hat{\mathcal{P}}_{\tau}(2 n)$. Thus $\hat{\mathcal{P}}_{\tau}(2 n)$ is dense in $\mathcal{P}_{\tau}(2 n)$.

Definition 2.1. For any $\tau>0$ and $\gamma \in \mathcal{P}_{\tau}(2 n)$, we define

$$
\nu_{\tau, \omega}(\gamma)=\operatorname{dim}_{\mathbf{C}} \operatorname{ker}_{\mathbf{C}}(\gamma(\tau)-\omega I), \quad \forall \omega \in \mathbf{U} .
$$

Definition 2.2 (cf. [Lo1]). For $\tau>0$ and $\omega \in \mathbf{U}$, given two paths $\gamma_{0}$ and $\gamma_{1} \in \mathcal{P}_{\tau}(2 n)$, if there exists a map $\delta \in C([0,1] \times[0, \tau], \operatorname{Sp}(2 n))$ such that $\delta(0, \cdot)=\gamma_{0}(\cdot), \delta(1, \cdot)=\gamma_{1}(\cdot), \delta(s, 0)=I$, and $\nu_{\tau, \omega}(\delta(s,)$.$) is constant for$ $0 \leq s \leq 1$, then $\gamma_{0}$ and $\gamma_{1}$ are $\omega$-homotopic on $[0, \tau]$ along $\delta(\cdot, \tau)$ and we write $\gamma_{0} \sim_{\omega} \gamma_{1}$. If $\gamma_{0} \sim_{\omega} \gamma_{1}$ on $[0, \tau]$ along $\delta(\cdot, \tau)$ for all $\omega \in \mathbf{U}$, then $\gamma_{0}$ and $\gamma_{1}$ are homotopic on $[0, \tau]$ along $\delta(\cdot, \tau)$ and we write $\gamma_{0} \sim \gamma_{1}$.

As well known, every $M \in \operatorname{Sp}(2 n)$ has its unique polar decomposition $M=A U$, where $A=\left(M M^{T}\right)^{1 / 2}$ is symmetric, positive definite, and symplectic, $U$ is orthogonal and symplectic. Therefore $U$ has the form $U=\left(\begin{array}{cc}u_{1} & -u_{2} \\ u_{2} & u_{1}\end{array}\right)$, where $u=u_{1}+\sqrt{-1} u_{2} \in \mathcal{L}\left(\mathbf{C}^{n}\right)$ is a unitary matrix. So for every path $\gamma \in \mathcal{P}_{\tau}(2 n)$ we can associate to $\gamma$ a path $u:[0, \tau] \rightarrow U(n)$ in the unitary group $U(n)$. If $\Delta(t)$ is any continuous real function satisfying det $u(t)=\exp (\sqrt{-1} \Delta(t))$, the difference $\Delta(\tau)-\Delta(0)$ depends only on $\gamma$ but 
not on the choice of the function $\Delta(t)$. Therefore we may define the rotation number of $\gamma$ on $[0, \tau]$ by

$$
\Delta_{\tau}(\gamma)=\Delta(\tau)-\Delta(0) \in \mathbf{R}
$$

Note that $\Delta_{\tau}: \mathcal{P}_{\tau}(2 n) \rightarrow \mathbf{R}$ is continuous.

Lemma 2.3. If $\gamma_{0}$ and $\gamma_{1} \in \mathcal{P}_{\tau}(2 n)$ possess common end point $\gamma_{0}(\tau)=$ $\gamma_{1}(\tau)$, then $\Delta_{\tau}\left(\gamma_{0}\right)=\Delta_{\tau}\left(\gamma_{1}\right)$ if and only if $\gamma_{0} \sim_{\omega} \gamma_{1}$ on $[0, \tau]$ for some $\omega \in \mathbf{U}$, if and only if $\gamma_{0} \sim \gamma_{1}$ on $[0, \tau]$.

This is a well known result as mentioned in [YS]. It can also be proved by using the function $\rho_{n}: \operatorname{Sp}(2 n) \rightarrow \mathbf{U}$ of $[\mathbf{S Z}]$ and the topological structure of the symplectic groups.

For any $\gamma \in \mathcal{P}_{\tau, \omega}^{*}(2 n)$, by Theorem 7.1 we can connect $\gamma(\tau)$ to $M_{n}^{-}$or $M_{n}^{+}$ by a path $\beta$ within $\operatorname{Sp}(2 n)_{\omega}^{*}$ and get a product path $\beta * \gamma$ defined by

$$
\beta * \gamma(t)= \begin{cases}\gamma(2 t), & \text { if } 0 \leq t \leq \frac{\tau}{2} \\ \beta(2 t-\tau), & \text { if } \frac{\tau}{2} \leq t \leq \tau\end{cases}
$$

Define

$$
k \equiv \frac{1}{\pi} \Delta_{\tau}(\beta * \gamma)
$$

Then $k$ is an integer, and by Theorem 7.1, it is independent of the choice of the path $\beta$. In this case we write $\gamma \in \mathcal{P}_{\tau, \omega}^{*}(2 n, k)$. These $\mathcal{P}_{\tau, \omega}^{*}(2 n, k)$ 's give a homotopy classification of $\mathcal{P}_{\tau, \omega}^{*}(2 n)$.

Definition 2.4. We define

$$
i_{\tau, \omega}(\gamma)=k, \quad \text { if } \gamma \in \mathcal{P}_{\tau, \omega}^{*}(2 n, k) .
$$

Thus for $\beta, \gamma \in \mathcal{P}_{\tau, \omega}^{*}(2 n), i_{\tau, \omega}(\beta)=i_{\tau, \omega}(\gamma)$ if and only if $\beta \sim_{\omega} \gamma$.

Next we study the degenerate paths. Fix $\omega \in \mathbf{U}$ and $\gamma \in \mathcal{P}_{\tau, \omega}^{0}(2 n)$. Using Theorem 7.5, there exists $P \in \operatorname{Sp}(2 n)$ such that (7.8) holds for $M=\gamma(\tau)$. Let $\Sigma_{0}(\omega, \gamma(\tau))$ be the subset of $\operatorname{Sp}(2 n)_{\omega}^{0}$ which contains all matrices $M$ satisfying

$$
\operatorname{dim}_{\mathbf{C}} \operatorname{ker}_{\mathbf{C}}(M-\omega I)>\nu_{\tau, \omega}(\gamma) .
$$

Let $\Sigma_{1}(\omega, \gamma(\tau))$ be the path connected component of $\operatorname{Sp}(2 n)_{\omega}^{0} \backslash \Sigma_{0}(\omega, \gamma(\tau))$ which contains $\gamma(\tau)$. For $\epsilon>0$, let $B_{\epsilon}(\gamma(\tau))$ be the open ball in $\operatorname{Sp}(2 n)$ centered at $\gamma(\tau)$ with radius $\epsilon$. Choose $\epsilon>0$ to be sufficiently small so that $\overline{B_{\epsilon}(\gamma(\tau))}$ is contractible and possesses no intersection with $\operatorname{Sp}(2 n)_{\omega}^{0} \backslash \Sigma_{1}(\omega, \gamma(\tau))$.

Let $\theta_{0} \in\left(0, \frac{\pi}{8 n}\right)$ and the integers $\left\{m_{1}, \ldots, m_{p+2 q}\right\}$ be the numbers defined in (7.9). For $s_{i} \in[-1,1]$ with $1 \leq i \leq p+2 q$, we define

$$
Q\left(s_{1}, \ldots, s_{p+2 q}\right) \equiv \gamma(\tau) P^{-1} R_{m_{1}}\left(s_{1} \theta_{0}\right) \cdots R_{m_{p+2 q}}\left(s_{p+2 q} \theta_{0}\right) P .
$$


Then for all $s_{i} \in[-1,1] \backslash\{0\}$ with $1 \leq i \leq p+2 q$, there hold

$$
\begin{cases}Q\left(s_{1}, \ldots, s_{p+2 q}\right) \in \operatorname{Sp}(2 n)_{\omega}^{*} \cap B_{\epsilon}(\gamma(\tau)), & 1 \leq k \leq p+2 q, \\ Q\left(s_{1}, \ldots, s_{p+2 q}\right) P^{-1} R_{m_{k}}\left(-s_{k} \theta_{0}\right) P \in \operatorname{Sp}(2 n)_{\omega}^{0}, & 1 \leq k \leq p+2 q,\end{cases}
$$

where the constant $a_{k}=1$ or 2 .

For $t_{0} \in(0, \tau)$, let $\rho \in C^{2}([0, \tau],[0,1])$ such that $\rho(t)=0$ for $0 \leq t \leq t_{0}$, $\dot{\rho}(t) \geq 0$ for $0 \leq t \leq \tau, \rho(\tau)=1$, and $\dot{\rho}(\tau)=0$. As in [Lo1], whenever $t_{0} \in(0, \tau)$ is sufficiently close to $\tau$, there holds $\gamma\left(\left[t_{0}, \tau\right]\right) \subset B_{\epsilon}(\gamma(\tau))$. For any $(s, t) \in[-1,1] \times[0, \tau]$, we define the rotational perturbation paths

$$
\gamma_{s}(t)=\gamma(t) P^{-1} R_{m_{1}}\left(s \rho(t) \theta_{0}\right) \cdots R_{m_{p+2 q}}\left(s \rho(t) \theta_{0}\right) P .
$$

They satisfy $\gamma_{s} \in \mathcal{P}_{\tau, \omega}^{*}(2 n)$ for $s \neq 0, \gamma_{0}=\gamma, \gamma_{s}(t)=\gamma(t)$ for $0 \leq t \leq t_{0}$, $\gamma_{ \pm 1}(\tau)=Q( \pm 1, \ldots, \pm 1), \gamma_{s}$ is continuous in $s \in[-1,1]$ and converges to $\gamma$ in $C^{0}$ as $s \rightarrow 0$, and

$$
\begin{cases}\gamma_{s}(t) \in B_{\epsilon}(\gamma(\tau)), & \forall(s, t) \in[0,1] \times\left[t_{0}, \tau\right] \\ \nu_{\tau, \omega}\left(\gamma_{s}\right)=0, & \forall s \in[-1,1] \backslash\{0\}, \\ i_{\tau, \omega}\left(\gamma_{s}\right)=i_{\tau, \omega}\left(\gamma_{s^{\prime}}\right), & \forall s, s^{\prime} \in[-1,1] \text { with } s s^{\prime}>0 .\end{cases}
$$

Note that if $\gamma \in \hat{\mathcal{P}}_{\tau, \omega}^{0}(2 n)$, we have $\gamma_{s} \in \hat{\mathcal{P}}_{\tau, \omega}^{*}(2 n)$ for $s \neq 0$ and $\gamma_{s} \rightarrow \gamma$ as $s \rightarrow 0$ in the topology of $\hat{\mathcal{P}}_{\tau}(2 n)$.

Theorem 2.5. For $\gamma \in \mathcal{P}_{\tau, \omega}^{0}(2 n)$, the rotational perturbation paths of $\gamma$ defined above by (2.6) satisfy

$$
i_{\tau, \omega}\left(\gamma_{1}\right)-i_{\tau, \omega}\left(\gamma_{-1}\right)=\nu_{\tau, \omega}(\gamma)
$$

Proof. Without loss of generality, we assume $\tau=1$. Note that this theorem has been proved in [Lo8] for $\omega=1$, and the case of $\omega=-1$ follows directly from the case of $\omega=1$ by the fact $D_{-1}(M)=D_{1}(-M)$ for all $M \in \operatorname{Sp}(2 n)$ with $D_{ \pm 1}$ defined in Section 7 . Here we only prove the theorem for $\omega \in \mathbf{U} \backslash \mathbf{R}$. Fix $\omega \in \mathbf{U} \backslash \mathbf{R}, \epsilon>0$, and $\gamma \in \mathcal{P}_{1, \omega}^{0}(2 n)$. Define $\gamma_{s}$ for $s \in[-1,1]$ by $(2.6)$. In the following we fix an $s \in(0,1]$.

For $0 \leq k \leq p+2 q$ define

$$
\alpha_{s, k}(t)=\gamma(t) \gamma(1)^{-1} Q\left(\mu_{1}(s, t), \ldots, \mu_{p+2 q}(s, t)\right), \quad \forall t \in[0,1],
$$

where $\mu_{i}(s, t)=\rho(t) s$ for $1 \leq i \leq k$ and $\mu_{i}(s, t)=-\rho(t) s$ for $k+1 \leq$ $i \leq p+2 q$. Then by a direct verification, we have $\alpha_{s, k} \in \mathcal{P}_{1, \omega}^{*}(2 n)$ for $0 \leq k \leq p+2 q, \alpha_{s, 0}=\gamma_{-s}, \alpha_{s, p+2 q}=\gamma_{s}$. We obtain $\nu_{1, \omega}(\gamma)=\sum_{k=1}^{p+2 q} a_{k}$. Assume the following equalities hold,

$$
i_{1, \omega}\left(\alpha_{s, k}\right)-i_{1, \omega}\left(\alpha_{s, k-1}\right)=a_{k}, \quad \text { for } 1 \leq k \leq p+2 q .
$$


Summing (2.10) up from $k=1$ to $k=p+2 q$, by Remark 7.4 and Theorem 7.5 we obtain (2.8). Therefore the proof of Theorem 2.5 is reduced to that of (2.10). Write $\alpha_{k}$ for $\alpha_{s, k}$ for notational simplicity.

Fix a $k \in\{1, \ldots, p+2 q\}$. Let $\eta(t)=\alpha_{k-1}(1) P^{-1} R_{m_{k}}\left(2 t s \theta_{0}\right) P$ for $t \in[0,1]$. Then by definition we have $\eta(0)=\alpha_{k-1}(1), \eta(1)=\alpha_{k}(1)$, $\eta(1 / 2)=\alpha_{k}(1) P^{-1} R_{m_{k}}\left(-s \theta_{0}\right) P \in \operatorname{Sp}(2 n)_{\omega}^{0}$, dimker $(\eta(1 / 2)-\omega I)=c_{k}$, and $\eta(t) \in \operatorname{Sp}(2 n)_{\omega}^{*}$ for $t \in[0,1] \backslash\{1 / 2\}$. By definition we obtain that $\left.\alpha_{k}\right|_{\left[t_{0}, 1\right]}$ is homotopic to $\left.\eta * \alpha_{k-1}\right|_{\left[t_{0}, 1\right]}$ with fixed end points. Therefore we obtain $\alpha_{k} \sim \eta * \alpha_{k-1}$ with fixed end points. Then (2.10) becomes

$$
i_{1, \omega}\left(\eta * \alpha_{k-1}\right)-i_{1, \omega}\left(\alpha_{k-1}\right)=a_{k} .
$$

When $a_{k}=2,(2.11)$ follows from the corresponding proof for Lemma 3 of [Lo1] or Theorem 3.2 of [Lo8] with the aid of Lemma 7.10. When $a_{k}=1$, (2.11) follows from the corresponding proof of Theorem 3.2 in [Lo8] with the aid of Theorem 7.2. Then the proof is complete.

Theorem 2.6. For $\gamma \in \mathcal{P}_{\tau, \omega}^{0}(2 n)$, there exists $\epsilon>0$ sufficiently small such that for any $\alpha \in \mathcal{P}_{\tau, \omega}^{*}(2 n) \cap B_{\epsilon}^{0}(\gamma)$ there holds

$$
i_{\tau, \omega}\left(\gamma_{-s}\right) \leq i_{\tau, \omega}(\alpha) \leq i_{\tau, \omega}\left(\gamma_{s}\right),
$$

where the paths $\gamma_{-s}$ and $\gamma_{s}$ are defined by $(2.6)$ for $s \in(0,1], B_{\epsilon}^{0}(\gamma)$ is the open ball centered at $\gamma$ with radius $\epsilon$ in $\mathcal{P}_{1}(2 n)$.

The proof of the Theorem 2.6 is postponed to Section 3. Then Theorem 2.6 yields:

Corollary 2.7. For $\gamma \in \mathcal{P}_{\tau, \omega}^{0}(2 n)$ and $s \in(0,1]$, the perturbation paths of $\gamma$ defined by (2.6) satisfy

$$
i_{\tau, \omega}\left(\gamma_{-s}\right)=\inf \left\{i_{\tau, \omega}(\alpha) \mid \alpha \in \mathcal{P}_{\tau}(2 n) \text { is } C^{0} \quad \text { sufficiently close to } \gamma\right\} \text {. }
$$

Specially for $s, s^{\prime} \in[-1,1] \backslash\{0\}$ there holds

$$
i_{\tau, \omega}\left(\gamma_{s}\right)=i_{\tau, \omega}\left(\gamma_{s^{\prime}}\right) \quad \text { if } s s^{\prime}>0 \text {. }
$$

Definition 2.8. For any $\gamma \in \mathcal{P}_{\tau, \omega}^{0}(2 n)$, we define

$$
i_{\tau, \omega}(\gamma)=i_{\tau, \omega}\left(\gamma_{-s}\right), \quad \forall 0<s \leq 1
$$

Proposition 2.9. Definition 2.8 of $i_{\tau, \omega}(\gamma)$ for any $\gamma \in \mathcal{P}_{\tau, \omega}^{0}(2 n)$ is well defined.

Proof. Without loss of generality, suppose $\tau=1$. Fix an $\omega \in \mathbf{U}$, a path $\gamma \in \mathcal{P}_{1, \omega}^{0}(2 n)$, and an $s \in(0,1]$. Define $\zeta_{s}(t)=Q(-s t, \ldots,-s t)$ for $t \in[0,1]$ by $(2.5)$. Then $\zeta_{s}(1)=\gamma_{-s}(1)$. Let $\psi_{-s}:[0,1] \rightarrow \operatorname{Sp}(2 n)_{\omega}^{*}$ be a path connecting $\gamma_{-s}(1)$ to $M_{n}^{+}$or $M_{n}^{-}$. Then the path $\gamma_{-s}$ is homotopic to the 
joint path $\zeta_{s} * \gamma$. Thus these two paths possess the same rotation numbers. Then we obtain

$$
i_{1, \omega}\left(\gamma_{s}\right)=\Delta_{1}\left(\psi_{-s} * \zeta_{s} * \gamma\right) / \pi=\Delta_{1}\left(\psi_{-s}\right) / \pi+\Delta_{1}\left(\zeta_{s}\right) / \pi+\Delta_{1}(\gamma) / \pi
$$

Thus $i_{1, \omega}(\gamma)$ is well defined.

Definition 2.10. For every path $\gamma \in \mathcal{P}_{\tau}(2 n)$, Definitions 2.1, 2.4, and 2.8 assign a pair of integers to $\gamma$ :

$$
\left(i_{\tau, \omega}(\gamma), \nu_{\tau, \omega}(\gamma)\right) \in \mathbf{Z} \times\{0, \ldots, 2 n\} .
$$

This pair of integers is called the $\omega$-index of $\gamma$. We call $i_{\tau, \omega}(\gamma)$ the $\omega$ rotation index of $\gamma$ and $\nu_{\tau}(\gamma)$ the $\omega$-nullity of $\gamma$.

Using $\diamond$-product defined in Section 7 , for any paths $\gamma_{j} \in \mathcal{P}_{\tau}\left(2 n_{j}\right)$ with $j=0$ and 1 , define $\gamma_{0} \diamond \gamma_{1}(t)=\gamma_{0}(t) \diamond \gamma_{1}(t)$ for all $t \in[0, \tau]$. For any $\tau>0$, $n \in \mathbf{N}$, and $k \in \mathbf{Z}$ using notations in Section 7 we define a sequence of zigzag standard paths $\alpha_{n, k, \tau}$ in $\mathcal{P}_{\tau}(2 n)$ as follows.

$$
\begin{aligned}
\phi_{\theta, \tau}(t) & =R(\theta t / \tau), \quad \forall t \in[0, \tau], \\
\alpha_{1,0, \tau}(t) & =D(1+t / \tau), \quad \forall t \in[0, \tau], \\
\alpha_{n, k, \tau} & =\left[\left(D(2) \phi_{k \pi, \tau}\right) * \hat{\alpha}_{1,0, \tau}\right] \diamond\left(\hat{\alpha}_{1,0, \tau}\right)^{\diamond(n-1)} .
\end{aligned}
$$

Then there hold $\alpha_{n, k, \tau} \in \mathcal{P}_{\tau, \omega}^{*}(2 n), i_{\tau, \omega}\left(\alpha_{n, k, \tau}\right)=k$ for all $\omega \in \mathbf{U}$ and $\alpha_{n, k, \tau}(\tau)=M_{n}^{ \pm}$if $(-1)^{k}= \pm 1$. The following theorem gives a characterization of our $\omega$-index theory.

Theorem 2.11. For any $\tau>0$ and $\omega=\exp (\theta \sqrt{-1}) \in \mathbf{U}$, there exists a unique function $i_{\tau, \omega}: \cup_{n \in \mathbf{N}} \mathcal{P}_{\tau}(2 n) \rightarrow \mathbf{Z}$ satisfying the following five axioms:

$1^{\circ}$ ( Homotopy invariant). For $\gamma_{0}$ and $\gamma_{1} \in \mathcal{P}_{\tau}(2 n)$, if $\gamma_{0} \sim_{\omega} \gamma_{1}$ on $[0, \tau]$, then

$$
i_{\tau, \omega}\left(\gamma_{0}\right)=i_{\tau, \omega}\left(\gamma_{1}\right)
$$

$2^{\circ}$ (Symplectic additivity). For any $\gamma_{i} \in \mathcal{P}_{\tau}\left(2 n_{i}\right)$ with $i=0$ and 1 , there holds

$$
i_{\tau, \omega}\left(\gamma_{0} \diamond \gamma_{1}\right)=i_{\tau, \omega}\left(\gamma_{0}\right)+i_{\tau, \omega}\left(\gamma_{1}\right)
$$

$3^{\circ}$ (Clockwise continuity). For any $\gamma \in \mathcal{P}_{\tau}(2 n)$ and $\omega \in \mathbf{U}$ satisfying $\gamma(\tau)=N_{1}(\omega, B)$ defined in Section 7 , there exists a $\theta_{0}>0$ such that

$$
i_{\tau, \omega}\left(\left(\gamma(\tau) \phi_{-\theta, \tau}\right) * \gamma\right)=i_{\tau, \omega}(\gamma), \quad \forall 0<\theta \leq \theta_{0} .
$$

$4^{\circ}$ ( Counterclockwise jumping). For any $\gamma \in \mathcal{P}_{\tau}(2 n)$ and $\omega \in \mathbf{U}$ satisfying $\gamma(\tau)=N_{1}(\omega, B)$ defined in Section 7 , there exists a $\theta_{0}>0$ such that

$$
i_{\tau, \omega}\left(\left(\gamma(\tau) \phi_{\theta, \tau}\right) * \gamma\right)=i_{\tau, \omega}(\gamma)+1, \quad \forall 0<\theta \leq \theta_{0} .
$$

$5^{\circ}$ ( Normality). There holds

$$
i_{\tau, \omega}\left(\alpha_{1,0, \tau}\right)=0
$$


Proof. When $\omega=1$, this theorem is proved in [Lo8]. From the fact $D_{-1}(M)$ $=D_{1}(-M)$ for all $M \in \operatorname{Sp}(2 n)$, the case of $\omega=-1$ follows from that of $\omega=1$. In the following we fix an $\omega \in \mathbf{U} \backslash \mathbf{R}$ with $\omega=\exp (\theta \sqrt{-1})$ and only prove the theorem for this case. Without loss of generality we suppose $\tau=1$. By our above discussions, similar to the proof in [Lo8], the index function $i_{1, \omega}: \cup_{n \in \mathbf{N}} \mathcal{P}_{1}(2 n) \rightarrow \mathbf{Z}$ defined by Definition 2.10 satisfies these five axioms.

Suppose we have another index function $\mu: \cup_{n \in \mathbf{N}} \mathcal{P}_{1}(2 n) \rightarrow \mathbf{Z}$ satifying these five conditions. It suffices to prove $\mu=i_{1, \omega}$ on $\mathcal{P}_{1}(2 n)$. Let $M=\gamma(1)$ in Corollary 7.11, we obtain a path $f \in \Omega_{\omega}^{0}(\gamma(1))$ with $f(0)=\gamma(1)$ and $f(1)$ is given by (7.14). Choose $\gamma_{j} \in \mathcal{P}_{1}(2)$ with $\gamma_{j}=M_{j}$ for $0 \leq j \leq n$ such that $\sum_{j=1}^{n} i_{1, \omega}\left(\gamma_{j}\right)=i_{1, \omega}(\gamma)$. Then by $2^{o}$, it suffices to prove $\mu=i_{1, \omega}$ on every path $\gamma$ in $\mathcal{P}_{1}(2)$ with $\gamma(1)$ possessing the form $R(\theta), R(2 \pi-\theta), D(2)$ or $D(-2)$. Thus by $3^{\circ}$, the value of $\mu$ on $\mathcal{P}_{1}(2 n)$ is completely determined by the value of $\mu$ on $\mathcal{P}_{1, \omega}^{*}(2)$. By $1^{o}$ these values are completely determined by $\mu$ on the standard paths $\alpha_{1, k, 1}$ 's. By $3^{o}, 4^{o}$ and $5^{o}$ we obtain $\mu\left(\alpha_{1, k, 1}\right)=k$ for all $k \in \mathbf{Z}$. Thus $\mu(\gamma)=i_{1, \omega}(\gamma)$ holds for every $\gamma \in \mathcal{P}_{1}(2 n)$. This completes the proof.

Remark 2.12. It is easy to construct examples to show that the five axioms in Theorem 2.11 are independent from each other. Theorem 1.2 is a direct consequences of Theorem 2.11. Note that when $\omega=1$ the $\omega$-inedx theory defined above coincides with the Maslov-type index theory defined in $[\mathbf{C Z}],[\mathbf{L Z}],[\mathbf{L o 1}],[\mathbf{L o 8}]$, and [Vi2]. Thus when $\omega=1$, we simply write $\left(i_{\tau}(\gamma), \nu_{\tau}(\gamma)\right)=\left(i_{\tau, 1}(\gamma), \nu_{\tau, 1}(\gamma)\right)$.

Theorem 2.13. (Inverse homotopy invariant.) For any two paths $\gamma_{0}$ and $\gamma_{1} \in \mathcal{P}_{\tau}(2 n)$ with $i_{\tau, \omega}\left(\gamma_{0}\right)=i_{\tau, \omega}\left(\gamma_{1}\right)$, suppose that there exists a continuous path $h:[0,1] \rightarrow \operatorname{Sp}(2 n)$ such that $h(0)=\gamma_{0}(\tau), h(1)=\gamma_{1}(\tau)$, and $\operatorname{dim}_{\mathbf{C}} \operatorname{ker}_{\mathbf{C}}(h(s)-\omega I)=\nu_{\tau, \omega}\left(\gamma_{0}\right)$ for all $s \in[0,1]$. Then $\gamma_{0} \sim_{\omega} \gamma_{1}$ on $[0, \tau]$ along $h$.

The proof of this theorem is similar to that of Theorem 6.4 in $[\mathbf{L o 8}]$ and thus is omitted.

Remark 2.14. For any end point free curve $f \in C([a, b], \operatorname{Sp}(2 n))$, choose $\gamma \in \mathcal{P}_{1}(2 n)$ so that $\gamma(1)=f(a)$. Define $\eta(t)=\gamma(2 t)$ for $t \in[0,1 / 2]$, and $\eta(t)=f(a+(2 t-1)(b-a))$ for $t \in[1 / 2,1]$. Define

$$
i_{\omega}(f)=i_{1, \omega}(\eta)-i_{1, \omega}(\gamma)
$$

By our above study of the index $i_{\tau, \omega}$ it is easy to see that $i_{\omega}(f)$ only depends on $f$ itself. Similar to Theorem 2.11, an axiom characterization of this index can be given. 


\section{Morse indices and $\omega$-indices.}

In this secion we study the relationship between the $\omega$-indices of the linear Hamiltonian system (1.2) and the Morse indices of the corresponding functionals defined on the truncated loop space, and prove Theorem 2.6.

For $\tau>0$, define $L_{\tau}$ to be the Hilbert space $L^{2}\left([0, \tau], \mathbf{C}^{2 n}\right)$ with the usual $L^{2}$ inner product $\langle\cdot, \cdot\rangle_{L_{\tau}}$, and $E_{\tau}=W^{1,2}\left(S_{\tau}, \mathbf{C}^{2 n}\right)$ with the usual $W^{1,2}$ norm as a subspace of $L_{\tau}$. For $\omega=\exp (\theta \sqrt{-1}) \in \mathbf{U}$, define $E_{\tau}^{\omega}$ to be the subspace $\left\{y \in E_{\tau} \mid y(\tau)=\omega y(0)\right\}$ of $L_{\tau}$. Any $y \in E_{\tau}^{\omega}$ has the form

$$
y(t)=\sum_{k \in \mathbf{Z}} e^{\sqrt{-1}(\theta+2 k \pi) t / \tau} \xi_{k}, \quad \xi_{k} \in \mathbf{C}^{2 n},
$$

where there holds

$$
\sum_{k \in \mathbf{Z}}\left((\theta+2 k \pi)^{2}+1\right)\left|\xi_{k}\right|^{2}<+\infty
$$

Define $E_{\tau, k}^{\omega}=E_{\tau, k}^{\omega,+} \oplus E_{\tau, k}^{\omega,-}$ with

$$
E_{\tau, k}^{\omega, \pm}=e^{\sqrt{-1}(\theta+2 k \pi) t / \tau}(J \pm \sqrt{-1} I) \mathbf{R}^{2 n} .
$$

Then there holds $E_{\tau}^{\omega}=\oplus E_{\tau, k}^{\omega}$.

Define $A=-J \frac{d}{d t}: E_{\tau}^{\omega} \rightarrow L_{\tau}$. Then $A$ is continuous and symmetric, i.e. it satisfies

$$
\langle A x, y\rangle_{L_{\tau}}=\langle x, A y\rangle_{L_{\tau}}, \quad \forall x, y \in E_{\tau}^{\omega} .
$$

Viewing $A$ as from the subspace $E_{\tau}^{\omega}$ of $L_{\tau}$ to $L_{\tau}$, we have

$$
\sigma(A)=\left\{\lambda_{k}^{ \pm} \mid k \in \mathbf{Z}\right\}, \quad \lambda_{k}^{ \pm}= \pm \frac{\theta+2 k \pi}{\tau},
$$

each eigenvalue $\lambda_{k}^{ \pm}$of $A$ has multiplicity $2 n$ and the corresponding eigenspace is $E_{\tau, k}^{\omega, \pm}$.

Given $B \in C\left(S_{\tau}, \mathcal{L}_{s}\left(\mathbf{R}^{2 n}\right)\right) \subset C\left(S_{\tau}, \mathcal{L}_{s}\left(\mathbf{C}^{2 n}\right)\right)$, it induces a symmetric operator on $L_{\tau}$ by

$$
\langle B x, y\rangle_{L_{\tau}}=\int_{0}^{\tau} B(t) x(t) \cdot \overline{y(t)} d t, \quad \forall x, y \in L_{\tau} .
$$

We consider the linear Hamiltonian system (1.2) for $y \in E_{\tau}^{\omega}$. Then $\tau$ periodic solutions of (1.2) are one to one correspondent to critical points of the functional

$$
f_{\tau, \omega}(y)=\frac{1}{2}\langle(A-B) y, y\rangle_{L_{\tau}}, \quad \forall y \in E_{\tau}^{\omega} \subset L_{\tau} .
$$

Then $f_{\tau, \omega}: E_{\tau}^{\omega} \rightarrow \mathbf{R}$ is smooth in the topology of $L_{\tau}$.

Using the saddle point reduction method (cf. $[\mathbf{C h}]$ ) we obtain a subspace

$$
Z_{\tau}^{\omega}=\oplus_{|m| \leq m_{0}} E_{\tau, k}^{\omega}
$$


with a sufficiently large $m_{0} \in \mathbf{N}$ (cf. Section 4.2 .1 of $[\mathbf{C h}]$ ), an injection map $u_{\tau, \omega} \in C^{\infty}\left(Z_{\tau}^{\omega}, L_{\tau}\right)$, and a smooth functional $a_{\tau, \omega} \in C^{\infty}\left(Z_{\tau}^{\omega}, \mathbf{R}\right)$ defined by

$$
a_{\tau, \omega}(z)=f_{\tau, \omega}\left(u_{\tau, \omega}(z)\right), \quad \forall z \in Z_{\tau}^{\omega} .
$$

Note that the origin of $Z_{\tau}^{\omega}$ as a critical point of $a_{\tau, \omega}$ corresponds to the origin of $E_{\tau}^{\omega}$ as a critical point of $f_{\tau, \omega}$. Denote by $m_{\tau, \omega}^{*}$ for $*=+, 0$, and - , the positive, null, and negative Morse indices of the functional $a_{\tau, \omega}$ at the origin respectively. Denote by $2 d_{\tau, \omega}=\operatorname{dim}_{\mathbf{C}} Z_{\tau}^{\omega}$.

Theorem 3.1. Let $\gamma \in \hat{\mathcal{P}}_{\tau}(2 n)$ be the fundamental solution of (1.2) with $B(t)$ given above. For any $\omega \in \mathbf{U}$, there hold

$$
\begin{aligned}
& m_{\tau, \omega}^{-}=d_{\tau, \omega}+i_{\tau, \omega}(\gamma) \\
& m_{\tau, \omega}^{0}=\nu_{\tau, \omega}(\gamma) \\
& m_{\tau, \omega}^{+}=d_{\tau, \omega}-i_{\tau, \omega}(\gamma)-\nu_{\tau, \omega}(\gamma) .
\end{aligned}
$$

Proof. For the case of $\omega=1$, this theorem was proved in $[\mathbf{C Z}],[\mathbf{L Z}],[\mathbf{L o 1}]$, and $[\mathbf{L o 8}]$. The case of $\omega=-1$ can be proved similarly and is left to the readers. Thus we fix an $\omega \in \mathbf{U} \backslash \mathbf{R}$ and prove the theorem for this case only. Without loss of generality, we suppose $\tau=1$.

Step 1. The case of $\gamma \in \hat{\mathcal{P}}_{\tau, \omega}^{*}(2 n)$.

For $k \in \mathbf{Z}$ define

$$
B_{k}=\left(k \pi I_{2}\right) \diamond O_{2 n-2}, \quad \beta_{k}(t)=\exp (t k \pi J) \diamond I_{2 n-2},
$$

where $O_{m}$ is the $m \times m$ zero matrix. Then $\beta_{k} \sim_{\omega} \hat{\alpha}_{n, k, 1}$ for all $k \in \mathbf{Z}$. Therefore there exists a $k \in \mathbf{Z}$ such that $\beta_{k} \sim_{\omega} \gamma$. By an analogue of Lemma 4.2 of [Lo8] for $Z_{1}^{\omega}$ and Definition 2.4, it suffices to prove (3.6)-(3.8) for $\gamma=\beta_{k}$ with any $k \in \mathbf{Z}$.

Fix $k \in \mathbf{Z}$. Define $B(t) \equiv B_{k}$ of (3.9) in (1.2). Since $B_{k}$ is a symmetric constant matrix, the corresponding functional $a_{\tau, \omega}$ satisfies (cf. Theorem 3.2 .1 of $[\mathbf{L o} 4])$

$$
a_{\tau, \omega}(z)=\frac{1}{2}\left\langle\left(A-B_{k}\right) z, z\right\rangle_{L_{\tau}}, \quad \forall z \in Z^{\omega} .
$$

Thus $a_{\tau, \omega}^{\prime \prime}(0)=A-B_{k}$. Denote its Morse indices by $m_{k}^{*}$ for $*=+, 0$, and -. We obtain (3.7) by the definition of $\beta_{k}$.

In order to verify (3.6) and (3.8), we consider properties of $\left.\left(A-B_{k}\right)\right|_{E_{\tau, m}^{\omega}}$. Write $\omega=e^{\sqrt{-1} \theta}$ with $\theta \in(0, \pi) \cup(\pi, 2 \pi)$. For any $m \in \mathbf{Z}$, let $\lambda_{m}=\theta+2 m \pi$. For $\zeta^{ \pm}=\left(\xi^{ \pm}, \eta^{ \pm}\right) \in \mathbf{R}^{2} \times \mathbf{R}^{2 n-2}$ we have

$$
z^{ \pm}=e^{\sqrt{-1} \lambda_{m} t}(J \pm \sqrt{-1} I) \zeta^{ \pm} \in E_{m}^{\omega, \pm} .
$$


Then there holds

$$
\begin{aligned}
& \left\langle\left(A-B_{k}\right)\left(z^{+}+z^{-}\right),\left(z^{+}+z^{-}\right)\right\rangle_{L_{\tau}} \\
& =2\left(\lambda_{m}-k \pi\right)\left|\xi^{+}\right|^{2}+2 \lambda_{m}\left|\eta^{+}\right|^{2}-2\left(\lambda_{m}+k \pi\right)\left|\xi^{-}\right|^{2}-2 \lambda_{m}\left|\eta^{-}\right|^{2} .
\end{aligned}
$$

Thus $A-B_{k}$ possesses the following four eigenvalues:

$$
\left\{\begin{array}{l}
\Lambda_{1}=2\left(\lambda_{m}-k \pi\right)=4 \pi\left(m+\frac{\theta}{2 \pi}-\frac{k}{2}\right), \\
\Lambda_{2}=2 \lambda_{m}=4 \pi\left(m+\frac{\theta}{2 \pi}\right) \\
\Lambda_{3}=-2\left(\lambda_{m}+k \pi\right)=-4 \pi\left(m+\frac{\theta}{2 \pi}+\frac{k}{2}\right), \\
\Lambda_{4}=-2 \lambda_{m}=-4 \pi\left(m+\frac{\theta}{2 \pi}\right),
\end{array}\right.
$$

where $\Lambda_{1}$ and $\Lambda_{3}$ are double eigenvalues, $\Lambda_{2}$ and $\Lambda_{4}$ are $(2 n-2)$-fold eigenvalues. Since $\beta_{k}$ is nondegenerate, there hold

$$
-\frac{\theta}{2 \pi} \pm \frac{k}{2} \text { and } \quad-\frac{\theta}{2 \pi} \notin \mathbf{Z} .
$$

Now we distinguish the study in three cases. Suppose $k>0$. By (3.12) there hold

$$
\begin{aligned}
& \Lambda_{1}<0, \quad \Lambda_{2}<0, \quad \Lambda_{3}>0, \quad \Lambda_{4}>0, \quad \text { if } m<-\frac{\theta}{2 \pi}-\frac{k}{2}, \\
& \Lambda_{1}<0, \quad \Lambda_{2}<0, \quad \Lambda_{3}<0, \quad \Lambda_{4}>0, \quad \text { if }-\frac{\theta}{2 \pi}-\frac{k}{2}<m<-\frac{\theta}{2 \pi}, \\
& \Lambda_{1}<0, \quad \Lambda_{2}>0, \quad \Lambda_{3}<0, \quad \Lambda_{4}<0, \quad \text { if }-\frac{\theta}{2 \pi}<m<-\frac{\theta}{2 \pi}+\frac{k}{2}, \\
& \Lambda_{1}>0, \quad \Lambda_{2}>0, \quad \Lambda_{3}<0, \quad \Lambda_{4}<0, \quad \text { if }-\frac{\theta}{2 \pi}+\frac{k}{2}<m .
\end{aligned}
$$

From $-\theta /(2 \pi) \pm(k / 2) \notin \mathbf{Z}$ we obtain

$$
\#\left\{\mathbf{Z} \cap\left(-\frac{\theta}{2 \pi}-\frac{k}{2},-\frac{\theta}{2 \pi}+\frac{k}{2}\right)\right\}=k .
$$

Summing up total numbers of positive and negative eigenvalues respectively for all $m \in\left[-m_{0}, m_{0}\right]$ defined in (3.4) we obtain

$$
m_{k}^{-}=d_{1, \omega}+k, \quad m_{k}^{+}=d_{1, \omega}-k .
$$

By similar computations, when $k<0$, we get (3.13), and when $k=0$, we obtain $m_{0}^{-}=d_{1, \omega}=m_{0}^{+}$. This completes the proof of Step 1 .

Step 2. The case of $\gamma \in \hat{\mathcal{P}}_{\tau, \omega}^{0}(2 n)$.

Similar to the proof in $[\mathbf{L o 8}]$ for $\omega=1$ we obtain

$$
m^{0}=\operatorname{dim}_{\mathbf{C}} \operatorname{ker}_{\mathbf{C}} a_{1, \omega}^{\prime \prime}(0)=\operatorname{dim}_{\mathbf{C}} \operatorname{ker}_{\mathbf{C}}(\gamma(1)-\omega I)=\nu_{1, \omega}(\gamma) .
$$

Define the perturbation paths $\gamma_{s}$ for $s \in[-1,1]$ by $(2.6)$. Define $B_{s}(t)=$ $-J \dot{\gamma}_{s}(t) \gamma_{s}(t)^{-1}$ for $(s, t) \in[-1,1] \times[0,1]$. Denote the functionals and maps on $E_{1}^{\omega}$ and the truncated space $Z_{1}^{\omega}$ corresponding to $B_{s}$ by $a_{s}=f_{s} \circ u_{s}$. Here $a_{0}=a_{1, \omega}$. Since $\gamma_{s} \rightarrow \gamma$ in $C^{1}$ as $s \rightarrow 0$, we obtain $B_{s} \rightarrow B$ in $C^{0}$ 
as $s \rightarrow 0$. Therefore by choosing $2 d_{1, \omega}=\operatorname{dim}_{\mathbf{C}} Z_{1}^{\omega}$ to be large enough, the space $Z_{1}^{\omega}$ can be defined uniformly for all $s \in[-1,1]$. Denote by $m_{s}^{*}$ for $*=+, 0$, and - . The origin $z=0$ is a nondegenerate isolated critical point of $a_{s}$ when $s \neq 0$. By Step 1, Theorem 3.1 holds for $B_{s}$ when $s \neq 0$, i.e.

$m_{s}^{-}=d_{1, \omega}+i_{1, \omega}\left(\gamma_{s}\right), \quad m_{s}^{0}=0, \quad m_{s}^{+}=d_{1, \omega}-i_{1, \omega}\left(\gamma_{s}\right), \quad$ if $s \in[-1,1] \backslash\{0\}$.

We also have $a_{s}$ converges to $a$ in $C^{2}$ as $s \rightarrow 0$. When $|s|>0$ is sufficiently small, the matrix $a_{s}^{\prime \prime}(0)$ is a small perturbation of $a^{\prime \prime}(0)$. Thus in this case there hold $m^{+} \leq m_{s}^{+}$and $m^{-} \leq m_{-s}^{-}$. So when $0<s \leq 1$ and sufficiently close to 0 there hold

$$
\begin{aligned}
& m^{+} \leq m_{s}^{+}=d_{1, \omega}-i_{1, \omega}\left(\gamma_{s}\right), \\
& m^{-} \leq m_{-s}^{-}=d_{1, \omega}+i_{1, \omega}\left(\gamma_{-s}\right) .
\end{aligned}
$$

By Theorem 2.5, the above (3.15) can be rewritten into

$$
m^{+} \leq d_{1, \omega}-i_{1, \omega}\left(\gamma_{s}\right)=d_{1, \omega}-i_{1, \omega}\left(\gamma_{-s}\right)-\nu_{1, \omega}(\gamma) .
$$

Note that $\operatorname{dim} Z_{1}^{\omega}=2 d_{1, \omega}$. We then obtain

$d_{1, \omega}-i_{1, \omega}\left(\gamma_{-s}\right) \leq 2 d_{1, \omega}-m^{-}=m^{+}+m^{0}=m^{+}+\nu_{1, \omega}(\gamma) \leq d_{1, \omega}-i_{1, \omega}\left(\gamma_{-s}\right)$.

Thus equalities must hold in (3.18) and they yield

$$
m^{-}=d_{1, \omega}+i_{1, \omega}\left(\gamma_{-s}\right) \quad \text { and } \quad m^{+}=d_{1, \omega}-i_{1, \omega}\left(\gamma_{-s}\right)-\nu_{1, \omega}(\gamma)
$$

Now (3.14) and (3.19) finish Step 2.

The proof of Theorem 3.1 is complete.

Now we can give:

Proof of Theorem 2.6. Without loss of generality we suppose $\tau=1$. We prove Theorem 2.6 in two steps.

Step 1. Firstly we suppose $\gamma \in \hat{\mathcal{P}}_{1}(2 n)$. Fix $s \in(0,1]$. We claim that there exists $\epsilon=\epsilon(\gamma)>0$ depending on $\gamma$ such that (2.12) holds for any path $\alpha \in \hat{\mathcal{P}}_{1, \omega}^{*}(2 n) \cap B_{\epsilon}^{1}(\gamma)$, where $B_{\epsilon}^{1}(\gamma)$ is the open ball centered at $\gamma$ with radius $\epsilon$ in $\hat{\mathcal{P}}_{1}(2 n)$.

We prove the left inequality of (2.12) indirectly by assuming that there exist $\alpha_{k} \in \hat{\mathcal{P}}_{1, \omega}^{*}(2 n)$ for $k \in \mathbf{N}$ such that $\alpha_{k}$ converges to $\gamma$ in $C^{1}$ as $k \rightarrow \infty$, and there holds

$$
i_{1, \omega}\left(\gamma_{-s}\right)>i_{1, \omega}\left(\alpha_{k}\right) .
$$

Denote by $B_{k}(t)=-J \dot{\alpha}_{k}(t) \alpha_{k}^{-1}(t)$. Then $B_{k} \rightarrow B$ in $C^{0}$. Therefore we can choose the truncated space $Z_{1}^{\omega}$ to be large enough for all the $B$ and $B_{k}$ to carry out the saddle point reduction. Let $2 d_{1, \omega}=\operatorname{dim} Z_{1}^{\omega}$. Denote by $m_{k}^{-}$, $m_{k}^{0}$, and $m_{k}^{+}$the Morse indices of the functional $a_{k}$ on $Z_{1}^{\omega}$ corresponding to $\alpha_{k}$, and by $m^{-}, m^{0}$, and $m^{+}$the Morse indices of the functional $a_{1, \omega}$ on $Z_{1}^{\omega}$ 
corresponding to $\gamma$. Then whenever $k$ is large enough, by Theorem 3.1 and (3.20) we obtain

$$
m^{-} \leq m_{k}^{-}=d_{1, \omega}+i_{1, \omega}\left(\alpha_{k}\right)<d_{1, \omega}+i_{1, \omega}\left(\gamma_{-s}\right) .
$$

Together with Theorem 3.1, we get the following contradiction

$$
d_{1, \omega}-i_{1, \omega}\left(\gamma_{-s}\right)=m^{+}+m^{0}=2 d_{1, \omega}-m^{-}>d_{1, \omega}-i_{1, \omega}\left(\gamma_{-s}\right) .
$$

This proves the left inequality in (2.12). The right inequality in (2.12) follows by a similar proof.

Step 2. Fix $\gamma \in \mathcal{P}_{1, \omega}^{0}(2 n)$, choose $\hat{\gamma} \in \hat{P}_{1}(2 n)$ such that $\hat{\gamma}(1)=\gamma(1)$ and $\hat{\gamma} \sim \gamma$. Specially this implies $\Delta_{1}(\hat{\gamma})=\Delta_{1}(\gamma)$. Thus by the construction in the proof of Theorem 2.5, we obtain $\hat{\gamma}_{s}(1)=\gamma_{s}(1)$ and $\hat{\gamma}_{s} \sim \gamma_{s}$ for all $s \in[-1,1]$ provided $\hat{\gamma}$ being sufficiently $C^{0}$ close to $\gamma$. By the homotopy invariant of the $\omega$-index for non-degenerate paths, we obtain

$$
i_{1, \omega}\left(\hat{\gamma}_{s}\right)=i_{1, \omega}\left(\gamma_{s}\right), \quad \nu_{1, \omega}\left(\hat{\gamma}_{s}\right)=\nu_{1, \omega}\left(\gamma_{s}\right), \quad \forall s \in[-1,1] \backslash\{0\} .
$$

For the constant $\epsilon=\epsilon(\hat{\gamma})>0$ given by the step 1 , let $B_{\epsilon / 2}^{0}(\gamma)$ be the open ball centered at $\gamma$ with radius $\epsilon / 2$ in $\mathcal{P}_{1}(2 n)$ under the $C([0,1], \operatorname{Sp}(2 n))$ topology. By further requiring this $\epsilon>0$ to be much smaller, we can assume

$$
\begin{array}{ll}
\left|\Delta_{1}(\hat{\gamma})-\Delta_{1}(\hat{\alpha})\right| \leq 1 / 2, \quad \forall \hat{\alpha} \in B_{\epsilon}^{1}(\hat{\gamma}), \\
\left|\Delta_{1}(\gamma)-\Delta_{1}(\alpha)\right| \leq 1 / 2, \quad \forall \alpha \in B_{\epsilon / 2}^{0}(\gamma) .
\end{array}
$$

Fix a path $\alpha \in \mathcal{P}_{1, \omega}^{*}(2 n) \cap B_{\epsilon / 2}^{0}(\gamma)$. Choose $\hat{\alpha} \in \hat{P}_{1, \omega}^{*}(2 n) \cap B_{\epsilon}^{1}(\hat{\gamma})$ such that $\hat{\alpha}(1)=\alpha(1)$. Then we obtain $\left|\Delta_{1}(\hat{\alpha})-\Delta_{1}(\alpha)\right| \leq 1$. This then implies $\Delta_{1}(\hat{\alpha})=\Delta_{1}(\alpha)$. By Lemma 2.3, this implies $\hat{\alpha} \sim \alpha$. Then by the homotopy invariant for the non-degenerate paths, we obtain $i_{1, \omega}(\hat{\alpha})=i_{1, \omega}(\alpha)$. Thus by (2.12) proved in Step 1 for $\hat{\gamma}$, we obtain (2.12) for $\gamma, \gamma_{-s}, \gamma_{s}$, and $\alpha$. The proof is complete.

Corollary 3.2. Fix $\omega \in \mathbf{U}$ and $\gamma \in \mathcal{P}_{\tau, \omega}^{0}(2 n)$. For any paths $\alpha$ and $\beta \in$ $\mathcal{P}_{\tau, \omega}^{*}(2 n)$ which are sufficiently $C^{0}$-close to $\gamma$, there hold

$$
\left|i_{\tau, \omega}(\beta)-i_{\tau, \omega}(\alpha)\right| \leq \nu_{\tau, \omega}(\gamma) .
$$

\section{Splitting numbers.}

Fix $n \in \mathbf{N}, \tau>0$ and a path $\gamma \in \mathcal{P}_{\tau}(2 n)$. In this section, we study the properties of the $\omega$-indices of $\gamma$,

$$
i_{\tau}(\omega) \equiv i_{\tau, \omega}(\gamma) \quad \text { and } \quad \nu_{\tau}(\omega) \equiv \nu_{\tau, \omega}(\gamma),
$$

as functions of $\omega \in \mathbf{U}$. By definition we obtain

$$
i_{\tau}(\omega)=i_{\tau}(\bar{\omega}) \quad \text { and } \quad \nu_{\tau}(\omega)=\nu_{\tau}(\bar{\omega}), \quad \forall \omega \in \mathbf{U} .
$$


Lemma 4.1. $\quad i_{\tau}$ is locally constant on $\mathbf{U} \backslash \sigma(\gamma(\tau))$, and thus is constant on each connected component of $\mathbf{U} \backslash \sigma(\gamma(\tau))$. There holds

$$
\nu_{\tau}(\omega)=0, \quad \forall \omega \in \mathbf{U} \backslash \sigma(\gamma(\tau)) .
$$

Proof. Note that $\mathbf{U} \cap \sigma(\gamma(\tau))$ contains at most $2 n$ points. For any $\omega_{0} \in \mathbf{U} \backslash \sigma(\gamma(\tau))$, let $\mathcal{N}\left(\omega_{0}\right)$ be an open connected neighborhood of $\omega_{0}$ in $\mathbf{U} \backslash \sigma(\gamma(\tau))$. By definition we obtain $\nu_{\tau}(\omega)=0$ for all $\omega \in \mathcal{N}\left(\omega_{0}\right)$. Thus (4.3) holds and $\gamma \in \mathcal{P}_{\tau, \omega}^{*}(2 n)$ for all $\omega \in \mathcal{N}\left(\omega_{0}\right)$. By Theorem 7.1, there is a path $\beta:[0,1] \rightarrow \mathcal{P}_{\tau, \omega_{0}}^{*}(2 n)$ connecting $\gamma(\tau)$ to $M_{n}^{+}$or $M_{n}^{-}$. By the compactness of the image of $\beta$ and the openness of $\operatorname{Sp}(2 n)_{\omega_{0}}^{*}$ in $\operatorname{Sp}(2 n)$, we can further require $\mathcal{N}\left(\omega_{0}\right)$ to be so small that $\beta$ is completely located within $\operatorname{Sp}(2 n)_{\omega}^{*}$ for all $\omega \in \mathcal{N}\left(\omega_{0}\right)$. Then by definition, this implies $i_{\tau}(\omega)=i_{\tau}\left(\omega_{0}\right)$ for all $\omega \in \mathcal{N}\left(\omega_{0}\right)$, and proves the lemma.

Corollary 4.2. The discontinuity points of $i_{\tau}(\cdot)$ and $\nu_{\tau}(\cdot)$ are contained in $\mathbf{U} \cap \sigma(\gamma(\tau))$.

Definition 4.3 (cf. [YS] and Section I.2 of [Ek]). Recall that for $M \in$ $\operatorname{Sp}(2 n)$ and $\omega \in \mathbf{U} \cap \sigma(M)$ being an $m$-fold eigenvalue, the Hermitian form $\langle\sqrt{-1} J \cdot, \cdot\rangle$, which is called the Krein form, is always nondegenerate on the invariant root vector space $E_{\omega}(M)=\operatorname{ker}_{\mathbf{C}}(M-\omega I)^{m}$, where $\langle\cdot, \cdot\rangle$ denotes the inner product in $\mathbf{C}^{2 n}$. Then $\omega$ is of Krein type $(p, q)$ with $p+q=m$ if the restriction of the Krein form on $E_{\omega}(M)$ has signature $(p, q) . \omega$ is Krein positive if it has Krein type $(p, 0)$, is Krein negative if it has Krein type $(0, q)$. If $\omega \in \mathbf{U} \backslash \sigma(M)$, we define the Krein type of $\omega$ by $(0,0)$.

It is well known that whenever 1 or $-1 \in \sigma(M)$, it must be a $2 p$-fold eigenvalue for some $p \in \mathbf{N}$ and have Krein type $(p, p)$. By direct computations we obtain:

Lemma 4.4. For any $M \in \operatorname{Sp}(2 n)$ and $\lambda \in \sigma(M)$, the algebraic and geometric multiplicities and the Krein type of $\lambda \in \sigma(N)$ for any $N \in \Omega^{0}(M)$ are the same as those of $M$.

We study next the splitting numbers $S_{M}^{ \pm}(\omega)$ defined in Theorem 1.3.

Lemma 4.5. The splitting numbers $S_{M}^{ \pm}(\omega)$ are well defined, i.e. is independent of the path $\gamma \in \mathcal{P}_{1}(2 n)$ appeared in (1.3). For $\omega \in \mathbf{U}$ and $M \in$ $\operatorname{Sp}(2 n), S_{N}^{ \pm}(\omega)$ are constant for any $N \in \Omega^{0}(M)$ of Definition 1.1.

Proof. We prove the theorem in two steps.

Step 1. Claim 1. $S_{M}^{ \pm}(\omega)$ is independent of the path $\gamma$ in its definition.

In fact, for $\gamma$ in (1.3) using (2.5) and a sufficiently small $a>0$, we define

$$
\xi_{a}(t)=\gamma(1) Q(-t a, \ldots,-t a), \quad \forall t \in[0,1] .
$$

Then we obtain

$$
i_{1, \omega}(\gamma)=i_{1, \omega}\left(\xi_{a} * \gamma\right),
$$


and by Theorem 7.1 there is a path $\beta_{0}:[0,1] \rightarrow \operatorname{Sp}(2 n)_{\omega}^{*}$ which connects $\xi_{\alpha}(1)$ to $M_{n}^{+}$or $M_{n}^{-}$defined in Section 7. Choose $\epsilon>0$ to be small enough such that $\lambda(s)=\exp (s \epsilon \sqrt{-1}) \omega \notin \sigma(M)$ for $0<s \leq 1$. By Lemma 4.1 there holds $i_{1, \lambda(s)}(\gamma)=$ constant for all $s \in(0,1]$. Thus by Theorem 7.1 , there exists a path $\beta_{\epsilon}:[0,1] \rightarrow \operatorname{Sp}(2 n)_{\lambda(1)}^{*}$ such that $\beta_{\epsilon}(0)=M$ and $\beta_{\epsilon}(1)=M_{n}^{+}$ or $M_{n}^{-}$. Thus by Definition 2.4 we obtain

$$
\begin{aligned}
S_{M}^{+}(\omega) & =i_{1, \lambda(1)}(\gamma)-i_{1, \omega}(\gamma) \\
& =\frac{1}{\pi} \Delta_{1}\left(\beta_{\epsilon} * \gamma\right)-\frac{1}{\pi} \Delta_{1}\left(\beta_{0} * \xi_{a} * \gamma\right) \\
& =\frac{1}{\pi}\left[\Delta_{1}\left(\beta_{\epsilon}\right)-\Delta_{1}\left(\beta_{0}\right)-\Delta_{1}\left(\xi_{a}\right)\right] .
\end{aligned}
$$

Thus $S_{M}^{+}(\omega)$ is independent of the choice of $\gamma$. By Theorem 7.1, this number is also independent of the choices of the above $a>0$ and paths $\beta_{\epsilon}$ and $\beta_{0}$, and then is completely determined by $M$ and $\omega$.

Step 2. Claim 2. $S_{M}^{ \pm}(\omega)=S_{N}^{ \pm}(\omega)$ for any $N \in \Omega^{0}(M)$.

In fact, by the definition of $N \in \Omega^{0}(M)$, there exists a path $\xi:[0,1] \rightarrow$ $\Omega(M)$ such that $\xi(0)=M$ and $\xi(1)=N$. Choose any $\gamma \in \mathcal{P}_{1}(2 n)$ such that $\gamma(1)=M$. Then by the definition of $\Omega(M)$, there holds $\gamma \sim \xi * \gamma$ on $[0,1]$ along $\xi$. Thus by Theorem 2.11, we obtain

$$
\begin{aligned}
S_{M}^{ \pm}(\omega) & =\lim _{\epsilon \rightarrow 0^{+}} i_{1, \exp ( \pm \epsilon \sqrt{-1}) \omega}(\gamma)-i_{1, \omega}(\gamma) \\
& =\lim _{\epsilon \rightarrow 0^{+}} i_{1, \exp ( \pm \epsilon \sqrt{-1}) \omega}(\xi * \gamma)-i_{1, \omega}(\xi * \gamma) \\
& =S_{N}^{ \pm}(\omega) .
\end{aligned}
$$

The proof is complete.

Note that Theorem 1.3 is contained in Lemma 4.5.

Lemma 4.6. For $M \in \operatorname{Sp}(2 n)$ and $\omega \in \mathbf{U}$, there hold

$$
\begin{aligned}
& S_{M}^{ \pm}(\omega)=0, \quad \text { if } \omega \notin \sigma(M) . \\
& S_{M}^{+}(\omega)=S_{M}^{-}(\bar{\omega}) .
\end{aligned}
$$

For any $M_{i} \in \operatorname{Sp}\left(2 n_{i}\right)$ with $i=0$ and 1 , there holds

$$
S_{M_{0} \diamond M_{1}}^{ \pm}(\omega)=S_{M_{0}}^{ \pm}(\omega)+S_{M_{1}}^{ \pm}(\omega), \quad \forall \omega \in \mathbf{U} .
$$

Proof. This is a direct consequence of Definition (1.3), Theorem 2.11, and Lemma 4.5.

In order to give a complete explanation of the splitting number, we introduce the concept of ultimate type of any $\omega \in \mathbf{U}$ as an eigenvalue of 
$M \in \mathrm{Sp}(2 n)$ as follows. We use the notation of basic normal forms introduced in Section 7.

Definition 4.7. A basic normal form matrix $M$ given by Definition 7.7 is trivial, if for sufficiently small $a>0, M R((t-1) a)^{\diamond n}$ possesses no eigenvalue on $\mathbf{U}$ for $t \in[0,1)$, and is nontrivial otherwise.

Note that by direct computations, $N_{1}(1,-1), N_{1}(-1,1), N_{2}(\omega, B)$ and $N_{2}(\bar{\omega}, B) \in \mathcal{M}_{\omega}^{1}(4)$ with $\omega=\exp (\theta \sqrt{-1}) \in \mathbf{U} \backslash \mathbf{R}$ and $\left(b_{2}-b_{3}\right) \sin \theta>0$ are trivial, and any other basic normal form matrix is nontrivial.

Definition 4.8. For any basic normal form $M \in \mathrm{Sp}(2 n)$ given by Definition 7.7 and $\omega \in \mathbf{U} \cap \sigma(M)$, we define the ultimate type $(p, q)$ of $\omega$ for $M$ to be its usual Krein type if $M$ is nontrivial, and to be $(0,0)$ if $M$ is trivial. For any $M \in \operatorname{Sp}(2 n)$, we define the ultimate type of $\omega$ for $M$ to be $(0,0)$ if $\omega \in \mathbf{U} \backslash \sigma(M)$. For any $M \in \operatorname{Sp}(2 n)$, by Theorem 7.8 there exists a $\diamond-$ product expansion (7.12) in the homotopy component $\Omega^{0}(M)$ of $M$ where each $M_{i}$ is a basic normal form for $1 \leq i \leq k$ and $\sigma\left(M_{0}\right) \cap \mathbf{U}=\emptyset$. Denote the ultimate type of $\omega$ for $M_{i}$ by $\left(p_{i}, q_{i}\right)$ for $0 \leq i \leq k$. Let $p=\sum_{i=0}^{k} p_{i}$ and $q=\sum_{i=0}^{k} q_{i}$. We define the ultimate type of $\omega$ for $M$ by $(p, q)$.

Proposition 4.9. The ultimate type of $\omega \in \mathbf{U}$ for $M$ is uniquely determined by $\omega$ and $M$, therefore is well defined. It is constant on $\Omega^{0}(M)$ for fixed $\omega \in \mathbf{U}$.

Proof. It suffices to notice that the $\diamond$-product (7.12) is uniquely determined by $M$ module the arranging order of $\left\{M_{1}, \ldots, M_{k}, M_{0}\right\}$ and the choice of the symplectic matrix $M_{0}$ satisfying $\omega \notin \sigma\left(M_{0}\right)$, and (7.12) holds for any $N \in \Omega^{0}(M)$. But these two factors make no change on the ultimate type defined above.

Lemma 4.10. For $\omega \in \mathbf{U}$ and $M \in \mathrm{Sp}(2 n)$, denote the Krein type and the ultimate type of $\omega$ for $M$ by $(P, Q)$ and $(p, q)$. Then there holds

$$
P-p=Q-q \geq 0 \text {. }
$$

Proof. Note that (4.7) holds for all basic normal forms of eigenvalues in $\mathbf{U}$. By the Krein Theorem (Theorem III.1.3 of [YS]), whenever the eigenvalue $\omega$ leaves $\mathbf{U}$ by a perturbation on $M$, both the Krein positive and negative type numbers of $\omega$ must decrease by the same integer. Therefore by the proof of Theorem 7.8, (4.7) must hold.

Theorem 4.11. For any $\omega \in \mathbf{U}$ and $M \in \operatorname{Sp}(2 n)$, there hold

$$
S_{M}^{+}(\omega)=p \quad \text { and } \quad S_{M}^{-}(\omega)=q,
$$

where $(p, q)$ is the ultimate type of $\omega$ for $M$.

Proof. By Lemmas 4.5, 4.6 and Theorem 7.8, it suffices to prove (4.8) for $M$ being the one of basic normal forms $N_{1}( \pm 1, B)$ with $B=1,0,-1, N_{1}(\omega, B)$ 
and $N_{2}(\omega, B) \in \mathcal{M}_{\omega}^{1}(2 n)$ for $\omega \in \mathbf{U} \backslash \mathbf{R}$ defined in Section 7 . We continue the proof according to different basic normal forms.

Case $1 . M=N_{1}(1, B)$ with $B= \pm 1$ or 0 .

Define $f_{B}(t)=N_{1}(1, t B)$ for $t \in[0,1]$. The matrix $M=N_{1}(1, B)$ is nontrivial when $B=1$ or 0 and possesses both the Krein type and the ultimate type $(1,1)$ of the eigenvalue 1 . The matrix $M=N(1,-1)$ is trivial, and possesses the Krein type $(1,1)$ and the ultimate type $(0,0)$ of the eigenvalue 1. Then using the $\mathbf{R}^{3}$ cylindrical coordinate representation introduced in [Lo2], it is easy to see that for any $\epsilon>0$ sufficiently small there hold

$$
\begin{aligned}
i_{1,1}\left(f_{B}\right) & =-1, \quad i_{1, \exp ( \pm \epsilon \sqrt{-1})}\left(f_{B}\right)=0, \quad \text { if } B=1 \text { or } 0, \\
\text { and } \quad i_{1,1}\left(f_{-1}\right) & =0, \quad i_{1, \exp ( \pm \epsilon \sqrt{-1})}\left(f_{-1}\right)=0 .
\end{aligned}
$$

Thus (4.8) holds when $\omega=1$.

Case 2. $M=N_{1}(-1, B)$ with $B= \pm 1$ or 0 .

The proof of the case of $\omega=-1$ is similar by using the $\mathbf{R}^{3}$-cylindrical coordinate representation of $[\mathbf{L o 2}]$ and is left to the readers.

Case 3. $M=R(\theta)$ or $R(2 \pi-\theta)$ with $\omega=e^{\theta \sqrt{-1}} \in \mathbf{U} \backslash \mathbf{R}$.

Fix $\omega=e^{\theta \sqrt{-1}} \in \mathbf{U} \backslash \mathbf{R}$. By Lemma 4.6 it suffices to prove (4.8) for $\theta \in(0,2 \pi)$ and $M=R(\theta)$. Fix a $\gamma \in \mathcal{P}_{\tau}(2)$ in (1.3).

When $\theta \in(0, \pi)$, note that $\sigma(R(\theta))$ consists of two simple eigenvalues $\omega$ and $\bar{\omega}$. By direct computation, $R(\theta)$ possess $(p, q)=(0,1)$ as the Krein type and ultimate type of $\omega$.

On the other hand, let $\lambda( \pm \epsilon)=\exp ( \pm \epsilon \sqrt{-1}) \omega$ with $\epsilon>0$ sufficiently small. When $\theta \in(0, \pi)$, the matrix $\gamma_{-1}(1)$ can be connected to $D(2)$ within $\operatorname{Sp}(2)_{\omega}^{+}$, where $\gamma_{-1}$ is the perturbation path of $\gamma$ defined by (2.6). Note also that the matrix $R(\theta)$ can be connected to $D(2)$ within $\operatorname{Sp}(2)_{\lambda(\epsilon)}^{+}$, and the matrix $R(\theta)$ can be connected to $D(-2)$ within $\operatorname{Sp}(2)_{\lambda(-\epsilon)}^{-}$. Thus there hold

$$
i_{1}(\lambda(-\epsilon))=i_{1}(\omega)+1, \quad i_{1}(\lambda(\epsilon))=i_{1}(\omega) .
$$

Therefore we obtain (4.8).

When $\theta \in(\pi, 2 \pi)$, similarly the matrix $\gamma_{-1}(1)$ can be connected to $D(-2)$ within $\operatorname{Sp}(2)_{\omega}^{-}$, the matrix $R(\theta)$ can be connected to $D(-2)$ within $\operatorname{Sp}(2)_{\lambda(\epsilon)}^{-}$, and the matrix $R(\theta)$ can be connected to $D(2)$ within $\operatorname{Sp}(2)_{\lambda(-\epsilon)}^{+}$. Thus (4.8) still hold.

Next we continue our study for $M=N_{2}(\omega, B) \in \mathcal{M}_{\omega}^{1}(4)$ with $\omega=e^{\theta \sqrt{-1}} \in$ $\mathbf{U} \backslash \mathbf{R}$ in two cases. By Lemma 4.6, it suffices to consider the case of $\omega \in$ $\sigma\left(N_{2}(\omega, B)\right)$ with $\theta \in(0, \pi) \cup(\pi, 2 \pi)$. In the following we always suppose $\theta \in(0, \pi)$. The case of $\theta \in(\pi, 2 \pi)$ can be studied similarly and is left to the readers. 
Case 4. $M=N_{2}(\omega, B) \in \mathcal{M}_{\omega}^{1}(4)$ and $\left(b_{2}-b_{3}\right) \sin \theta<0$.

Since $N_{2}(\omega, B)$ is nontrivial, by direct computation we obtain that $\omega$ as an eigenvalue of $M$ possesses both the Krein type and the ultimate type $(1,1)$.

Define

$$
\xi(t)=M R((t-1) \alpha)^{\diamond 2}, \quad \forall t \in[0,1],
$$

for some small $\alpha \in(0, \theta / 2]$. Note that there holds

$$
\operatorname{det}\left(\xi(t)-\lambda I_{4}\right)=\lambda^{4}-4 A_{\xi}(t) \lambda^{3}+B_{\xi}(t) \lambda^{2}-4 A_{\xi}(t) \lambda+1 .
$$

Then denoting $s=\sin \theta, c=\cos \theta, s_{1}=\sin ((t-1) \alpha)$ and $c_{1}=\cos ((t-1) \alpha)$ for $j=1$ and 2 there, we obtain that for sufficiently small $\alpha>0$ and $t \in[0,1)$,

$$
\begin{aligned}
D_{\omega}(\xi(t)) & =2 s_{1} c_{1} s\left(b_{2}-b_{3}\right)+o\left(\left|s_{1}\right|\right)>0, \\
4 A_{\xi}^{2}(t)+2-B_{\xi}(t) & =2 s_{1} c_{1} s\left(b_{2}-b_{3}\right)+o\left(\left|s_{1}\right|\right)>0,
\end{aligned}
$$

where $s_{1}=\sin ((t-1) \alpha)<0$. Here the condition $s\left(b_{2}-b_{3}\right)<0$ is crucial. Thus by Lemma 7.9, for $0 \leq t<1$ the matrix $\xi(t)$ possesses two pairs of simple eigenvalues $\left\{\lambda_{1}(t), \overline{\lambda_{1}(t)}, \lambda_{2}(t), \overline{\lambda_{2}(t)}\right\}$ such that for $j=1$ and $2, \lambda_{j} \in$ $C([0,1], \mathbf{U}), \lambda_{j}(1)=\omega, \lambda_{j}(t) \neq \omega$ and $\left|\lambda_{j}(t)-\omega\right|$ is sufficiently small provided $\alpha>0$ is small enough for $t \in[0,1)$. Denote by $\lambda_{j}(t)=\exp \left(\beta_{j}(t) \sqrt{-1}\right)$ with $\beta_{j} \in C([0,1], \mathbf{R})$ for $j=1$ and 2 , and $\beta_{1}(1)=\beta_{2}(1)=\theta$. Note that $\xi(t)$ is the fundamental solution of the system (1.2) with $B(t)=-J \dot{\xi}(t) \xi(t)^{-1}$ being negative definite in the sense of $[\mathbf{E k}]$. So When $0 \leq t<1, \lambda_{1}(t)$ and $\lambda_{2}(t)$ must have different Krein type. Without loss of generality, for $0 \leq t<1$ we assume that $\lambda_{1}(t)$ is Krein positive and $\lambda_{2}(t)$ is Krein negative. By the proof of Propositions I.3.2 of $[\mathbf{E k}]$, we obtain

$$
\beta_{1}(t)>\theta>\beta_{2}(t), \quad \forall 0 \leq t<1,
$$

and $\beta_{1}(t)$ and $\beta_{2}(t)$ are strictly decreasing and increasing respectively when $t$ increases to 1 .

By (4.12), we have $\xi(0) \in \operatorname{Sp}(4)_{\omega}^{-}$. Thus there is a path $\eta:[0,1] \rightarrow$ $\operatorname{Sp}(2 n)_{\omega}^{-}$connecting $\eta(0)=D(-2) \diamond D(2)$ to $\eta(1)=\xi(0)$. Denote by $\psi=$ $\alpha_{2,1,1}$ the standard zigzag path defined in Section 2 . Then $\psi$ connects $I_{4}$ to $D(-2) \diamond D(2)$ and there holds $i_{1, \lambda}(\psi)=1$ for all $\lambda \in \mathbf{U}$. Thus

$$
i_{1, \omega}(\eta * \psi)=1 \text {. }
$$

Define

$$
\gamma=\xi * \eta * \psi .
$$

Denote by $s$ and $c$ as in (4.12). For any $b \in[-\alpha, 0]$, let $s_{1}=\sin (-\alpha)$, $c_{1}=\cos (-\alpha), s_{2}=\sin b, c_{2}=\cos b$. By Lemma 7.10 we further require 
$\alpha>0$ to be so small such that for all $b \in[-\alpha, 0]$ there holds $(4.17)$

$D_{\omega}(M[R(-\alpha) \diamond R(b)])=2 s^{2}\left(2-c_{1}-c_{2}\right)+2 s^{2} s_{1} s_{2}-2\left(1+c^{2}\right)\left(1-c_{1}\right)\left(1-c_{2}\right)>0$.

Thus $M\left[R(-\alpha) \diamond I_{2}\right]$ and $M[R(-\alpha) \diamond R(-\alpha)]$ can be connected within $\operatorname{Sp}(4)_{\omega}^{*}$. This proves $\gamma_{-1} \sim_{\omega} \eta * \psi$, where $\gamma_{-1}$ is the perturbation path of $\gamma$ defined by (2.6). Thus

$$
i_{1, \omega}(\gamma)=i_{1, \omega}\left(\gamma_{-1}\right)=i_{1, \omega}(\eta * \psi)=1 .
$$

Recall that we have supposed $\theta \in(0, \pi)$. So we can fix $\epsilon>0$ sufficiently small so that for $\chi( \pm \epsilon)=e^{ \pm \epsilon \sqrt{-1}} \omega$ there hold

$$
\begin{gathered}
i_{1, \chi( \pm \epsilon)}(\gamma)=\lim _{s \rightarrow 0^{+}} i_{1, \exp ( \pm s \sqrt{-1}) \omega}(\gamma), \\
S(\omega) \equiv\{\exp (a \sqrt{-1}) \omega \in \mathbf{U}|| a \mid \leq \epsilon / 2\} \subset \mathbf{U} \backslash \mathbf{R} .
\end{gathered}
$$

Choose $r \in(0,1)$ close to 1 enough so that

$$
\sigma(\xi(t)) \subset S(\omega) \cup S(\bar{\omega}), \quad \forall t \in[r, 1] .
$$

Define $\hat{\xi}(t)=\xi(t r)$ for $t \in[0,1]$. Note that (4.21) implies

$$
i_{1, \chi( \pm \epsilon)}(\gamma)=i_{1, \chi( \pm \epsilon)}(\hat{\xi} * \eta * \psi)
$$

By (4.14) and the discussion there, $\lambda_{1}(r)$ and $\lambda_{2}(r)$ are Krein positive and negative respectively. By (4.20) and (4.21), both $\beta_{1}(r)$ and $\beta_{2}(r)$ are in $(0, \pi)$. Thus by Theorem 7.6 there exists $P \in \operatorname{Sp}(4)$ such that $P \xi(r) P^{-1}=$ $R\left(2 \pi-\beta_{1}(r)\right) \diamond R\left(\beta_{2}(r)\right)$. Let $p \in \mathcal{P}_{1}(4)$ satisfy $p(1)=P$. Define $\zeta(t)=$ $p(t) \xi(r) p(t)^{-1}$ for all $t \in[0,1]$. Then the conjugate path $\zeta$ connects $\xi(r)$ to $R\left(2 \pi-\beta_{1}(r)\right) \diamond R\left(\beta_{2}(r)\right)$. Define

$$
\begin{aligned}
f_{1}(t) & =R\left(t\left(2 \pi-\beta_{1}(r)\right)\right), \quad f_{2}(t)=R\left(t \beta_{2}(r)\right), \\
f(t) & =f_{1} \diamond f_{2}(t), \quad \forall t \in[0,1] .
\end{aligned}
$$

By (4.14), there holds $\beta_{1}(r)>\theta>\beta_{2}(r)$. Thus by Theorem 2.11 and a direct computation for paths in $\operatorname{Sp}(2)$, we obtain

$$
\begin{aligned}
i_{1, \omega}(f) & =1+0=1, \\
i_{1, \chi(\epsilon)}(f) & =2+0=2, \quad i_{1, \chi(-\epsilon)}(f)=1+1=2 .
\end{aligned}
$$

By (4.15) and the definitions of $\hat{\xi}$ and $\zeta$ we obtain

$$
i_{1, \omega}(\zeta * \hat{\xi} * \eta * \psi)=i_{1, \omega}(\eta * \psi)=1 .
$$

In Figure 4.1, this computation is intuitively explained via the $\mathbf{R}^{3}$-cylindrical representation of $\operatorname{Sp}(2)$ of [Lo2] on the plane $\{z=0\}$, where $f_{1}$ and $f_{2}$ are represented by two nearby paths with the same topological properties. Since $\zeta * \hat{\xi} * \eta * \psi(1)=f(1)$, by Theorem 2.13, (4.24), and (4.26), we obtain $\zeta * \hat{\xi} * \eta * \psi \sim f$ on $[0,1]$ with fixed end points. Since $\zeta$ is a conjugation 
path, this implies $\hat{\xi} * \eta * \psi \sim f$ on $[0,1]$ along $\zeta$. Then by Theorem 2.11 and (4.25) we obtain

$$
i_{1, \chi( \pm \epsilon)}(\hat{\xi} * \eta * \psi)=i_{1, \chi( \pm \epsilon)}(f)=2 .
$$

Together with (4.22) we obtain

$$
i_{1, \chi( \pm \epsilon)}(\gamma)=2 .
$$

By (4.18), (4.19), and (4.28), we obtain

$$
S_{M}^{+}(\omega)=S_{M}^{-}(\omega)=1,
$$

i.e. (4.8) holds in this case.

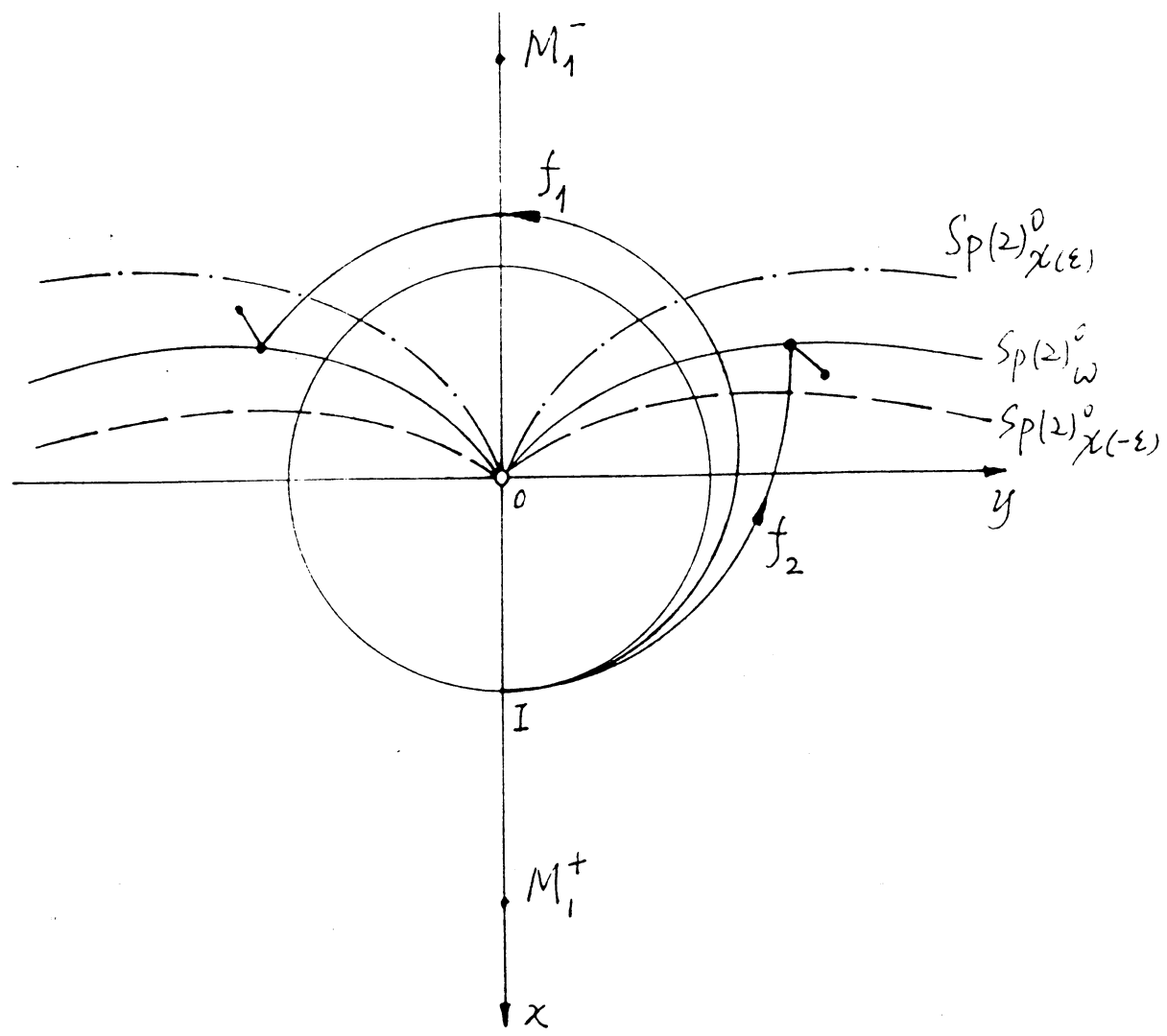

Figure 4.1.

Case 5. $M=N_{2}(\omega, B) \in \mathcal{M}_{\omega}^{1}(4)$ and $\left(b_{2}-b_{3}\right) \sin \theta>0$.

Since in this case $M$ is trivial, by direct computation we obtain that $\omega$ as an eigenvalue of $M$ possesses the Krein type $(1,1)$ and the ultimate type $(0,0)$. 
Define the path $\xi:[0,1] \rightarrow \operatorname{Sp}(4)$ by (4.10) for some small $\alpha \in(0, \theta / 2]$. By the notations of Case 4, we obtain that for sufficiently small $\alpha>0$ and $t \in[0,1)$ as in (4.12) and (4.13) there hold:

$$
D_{\omega}(\xi(t))<0, \quad 4 A_{\xi}^{2}(t)+2-B_{\xi}(t)<0,
$$

where $s_{1}=\sin ((t-1) \alpha)<0$. Here the condition $s\left(b_{2}-b_{3}\right)<0$ is crucial. By Lemma 7.9, this implies

$$
\sigma(\xi(t)) \cap(\mathbf{U} \cup \mathbf{R})=\emptyset, \quad \forall t \in[0,1) .
$$

Similar to our study in Case 1 , by (4.30) we obtain that $M_{2}^{+}$can be connected to $\xi(0)$ by a path $\eta:[0,1] \rightarrow \operatorname{Sp}(2 n)$ satisfying

$$
\sigma(\eta(t)) \cap \mathbf{U}=\emptyset, \quad \forall t \in[0,1] .
$$

Define $\psi=\alpha_{2,0,1}$ of Section 2. Then $\psi$ connects $I_{4}$ to $M_{2}^{+}$and satisfies $i_{1, \lambda}(\psi)=0$ for all $\lambda \in \mathbf{U}$. Together with (4.32) there holds

$$
i_{1, \omega}(\eta * \psi)=0 .
$$

Similar to the proof of (4.18), using Lemma 7.10, we get that for $\gamma=\xi * \eta * \psi$ there holds

$$
i_{1, \omega}(\gamma)=i_{1, \omega}(\eta * \psi)=0
$$

Fix $\epsilon>0$ so small that (4.19) holds for $\chi( \pm \epsilon)=e^{ \pm \epsilon \sqrt{-1}} \omega \in \mathbf{U} \backslash \mathbf{R}$. By (4.33) and (4.34), $\chi( \pm \epsilon) \notin \sigma(\gamma(t))$ for $0 \leq t \leq 1$. Since $\gamma$ passes through $M_{2}^{+}$, we obtain

$$
i_{1, \chi( \pm \epsilon)}(\gamma)=0
$$

Then together with (4.19) and (4.34) we obtain

$$
S_{M}^{+}(\omega)=S_{M}^{-}(\omega)=0,
$$

i.e. (4.8) holds in this case.

The proof is complete.

Now we obtain the following results immediately.

Corollary 4.12. If $\omega \in \mathbf{U} \cap \sigma(\gamma(\tau))$ is of Krein type $(p, q)$, there holds

$$
\lim _{\epsilon \rightarrow 0^{+}}\left(i_{\tau}\left(e^{\epsilon \sqrt{-1}} \omega\right)-i_{\tau}\left(e^{-\epsilon \sqrt{-1}} \omega\right)\right)=p-q .
$$

Corollary 4.13. For any $\omega \in \mathbf{U}$ and $M \in \mathrm{Sp}(2 n)$, there holds

$$
0 \leq S_{M}^{ \pm}(\omega) \leq \operatorname{dim}_{\mathbf{C}} \operatorname{ker}_{\mathbf{C}}(M-\omega I) \text {. }
$$

Corollary 4.14. The integer valued splitting number pair $\left(S_{M}^{+}(\omega), S_{M}^{-}(\omega)\right)$ defined for all $(\omega, M) \in \mathbf{U} \times \cup_{n \geq 1} \mathrm{Sp}(2 n)$ are uniquely determined by the following axioms:

$1^{\circ}$ (Homotopy invariant). $S_{M}^{ \pm}(\omega)=S_{N}^{ \pm}(\omega)$ for all $N \in \Omega^{0}(M)$. 
$2^{\circ}$ (Symplectic additivity). $S_{M_{1} \diamond M_{2}}^{ \pm}(\omega)=S_{M_{1}}^{ \pm}(\omega)+S_{M_{2}}^{ \pm}(\omega)$ for all $M_{i} \in$ $\operatorname{Sp}\left(2 n_{i}\right)$ with $i=1$ and 2 .

$3^{\circ}$ (Vanishing). $S_{M}^{ \pm}(\omega)=0$ if $\omega \notin \sigma(M)$.

$4^{\circ}$ (Normality). ( $\left.S_{M}^{+}(\omega), S_{M}^{-}(\omega)\right)$ coincides with the ultimate type of $\omega$ for $M$ when $M$ is any basic normal form given by Definition 7.7.

Remark 4.15. Our Theorem 4.11 and Corollary 4.14 give a complete understanding of the splitting numbers for all $M \in \operatorname{Sp}(2 n)$. Consequently, the ultimate type is also characterized by Corollary 4.14. Note that Example II on page 181 of [Bo] coincides with our Theorem 4.11. Since [Bo] uses the same $J$ as we do, the Theorem IV of [Bo] should be corrected by exchanging the positive and the negative Krein numbers.

\section{Bott-type formulae and the mean index.}

Based on the results of the previous sections, following the idea of [Bo], we now prove Theorems 1.4, 1.5 and 1.6 for the Maslov-type index theory.

Proof of Theorem 1.4. Fix $\tau>0$ and $B \in C\left(S_{\tau}, \mathcal{L}_{s}\left(\mathbf{R}^{2 n}\right)\right)$. Let $\gamma:[0,+\infty) \rightarrow$ $\operatorname{Sp}(2 n)$ be the fundamental solution of (1.2). Fix $k \in \mathbf{N}$. The bilinear form $\phi_{k \tau}$ on $E_{k \tau}^{1}$ corresponding to $f_{k \tau, 1}$ in Section 3 is given by

$$
\phi_{k \tau}(x, y)=\frac{1}{2}\langle(A-B) x, y\rangle_{L_{\tau}}, \quad \forall x, y \in \operatorname{dom} A=E_{k \tau}^{1} \subset L_{k \tau}^{1} .
$$

Two subspaces $F_{1}$ and $F_{2}$ of $E_{k \tau}^{1}$ are $\phi_{k \tau}$-orthogonal and is denoted by $F_{1} \perp F_{2}$, if there holds $\phi_{k \tau}(x, y)=0$ for all $x \in F_{1}$ and $y \in F_{2}$. We denote by $F_{1} \oplus F_{2}$ the $\phi_{k \tau}$-orthogonal product of $F_{1}$ and $F_{2}$. For $\omega \in \mathbf{U}$ define

$$
E_{k \tau}^{1}(\tau, \omega)=\left\{y \in E_{k \tau}^{1} \mid y(t+\tau)=\omega y(t), \forall t\right\} .
$$

In the following for notational simplicity we identify $E_{k \tau}^{1}(\tau, \omega)$ with $E_{\tau}^{\omega}$. For $0 \leq p \leq k$, define $\omega_{p}=\exp (2 p \pi / k \sqrt{-1})$. Then $\omega_{p}^{k}=1$. We claim that there hold

$$
\begin{aligned}
& E_{k \tau}^{1}\left(\tau, \omega_{p}\right) \perp E_{k \tau}^{1}\left(\tau, \omega_{q}\right), \quad \text { if } 0 \leq p \neq q \leq k, \\
& E_{k \tau}^{1}=\oplus_{\omega^{k}=1} E_{k \tau}^{1}(\tau, \omega) .
\end{aligned}
$$

In fact, for $x \in E_{k \tau}^{1}\left(\tau, \omega_{p}\right)$ and $y \in E_{k \tau}^{1}\left(\tau, \omega_{q}\right)$ with $0 \leq p \neq q \leq k$, we have

$$
\phi_{k \tau}(x, y)=\phi_{\tau}(x, y) \sum_{m=0}^{k-1}\left(\omega_{p} \bar{\omega}_{q}\right)^{m}=0 .
$$

Thus (5.1) holds, and then (5.2) follows. 
We consider the functional $f_{k \tau, 1}$ defined by (3.3). For any $\alpha_{i} \in \mathbf{C}$ and $y_{i} \in E_{k \tau}^{1}\left(\tau, \omega_{i}\right)$ with $\omega_{i}^{k}=1$ and $0 \leq i \leq k-1$, there holds

$$
f_{k \tau, 1}\left(\sum_{i=0}^{k-1} \alpha_{i} y_{i}\right)=\sum_{i=0}^{k-1} f_{k \tau, 1}\left(\alpha_{i} y_{i}\right)=k \sum_{i=0}^{k-1} f_{\tau, 1}\left(\alpha_{i} y_{i}\right) .
$$

This yields

$$
\left.f_{k \tau, 1}\right|_{E_{k \tau}^{1}}=\left.\sum_{i=0}^{k-1} f_{k \tau, 1}\right|_{E_{k \tau}^{1}\left(\tau, \omega_{i}\right)}=\left.k \sum_{i=0}^{k-1} f_{\tau, \omega_{i}}\right|_{E_{\tau}^{\omega_{i}}} .
$$

Now we carry out the saddle point reduction for $f_{k \tau, 1}$ on $E_{k \tau}^{1}$, and obtain the functional $a_{k \tau, 1}=f_{k \tau, 1} \circ u_{k \tau, 1}$ defined on $Z_{k \tau}^{1}$. Simultaneously this induces saddle point reductions for $f_{\tau, \omega_{i}}$ on $E_{k \tau}^{1}\left(\tau, \omega_{i}\right)$ for $0 \leq i \leq k-1$, and yields the functional $a_{\tau, 1}\left(\tau, \omega_{i}\right)=f_{\tau, 1} \circ u_{\tau, 1}\left(\tau, \omega_{i}\right)$ defined on $Z_{k \tau}^{1}(\tau, \omega)$. Denote the Morse indices of $a_{\tau, 1}\left(\tau, \omega_{i}\right)$ at the origin by $\hat{m}_{\tau}^{*}\left(\omega_{i}\right)$ for $*=+$, 0 , and - . Then these Morse indices coincide with the Morse indices $m_{\tau}^{*}\left(\omega_{i}\right)$ of the functional $a_{\tau, \omega_{i}}$ on $Z_{\tau}^{\omega}$ at the origin defined next to (3.5). Therefore from (5.2) and (5.3) we obtain

$$
m_{k \tau}^{*}(1)=\sum_{\omega^{k}=1} \hat{m}_{\tau}^{*}(\omega)=\sum_{\omega^{k}=1} m_{\tau}^{*}(\omega), \quad \text { for } *=+, 0,-
$$

Note that there holds

$$
d_{k \tau, 1}=\sum_{\omega^{k}=1} d_{\tau, \omega}
$$

Combining (5.4), (5.5), and Theorem 3.1, we have proved the special case of the Bott formula Theorem 1.4 for $\gamma \in \hat{\mathcal{P}}_{\tau}(2 n)$.

Now fix $\gamma \in \mathcal{P}_{\tau}(2 n)$. Choose $\beta \in \hat{P}_{\tau}(2 n)$ such that $\beta(\tau)=\gamma(\tau)$ and $\beta \sim \gamma$. We obtain $i_{\tau, \omega}(\beta)=i_{\tau, \omega}(\gamma)$ for all $\omega \in \mathbf{U}$. From $\beta \sim \gamma$ with fixed end points, this homotopy can be extended to $[0,1] \times[0, k \tau]$. By Theorem 2.11 , we then obtain $\beta^{k} \sim \gamma^{k}$. Thus $i_{k \tau}\left(\beta^{k}\right)=i_{k \tau}\left(\gamma^{k}\right)$ holds. Then the Bott-type formula for $\beta$ implies that for $\gamma$. This completes the proof of Theorem 1.4.

Remark 5.1. Theorem 1.4 can also be proved based on Theorem 2.11 of the $\omega$-index theory by reducing the path $\gamma \in \operatorname{Sp}(2 n)$ to paths in $\operatorname{Sp}(2)$, and then by direct verifications without using the above analytic method of $R$. Bott.

Fix $\tau>0$ and $\gamma \in \mathcal{P}_{\tau}$ in the rest of this section. Let $i_{k \tau}(\omega)=i_{k \tau, \omega}(\tilde{\gamma})$ and $\nu_{k \tau}(\omega)=\nu_{k \tau, \omega}(\tilde{\gamma})$ for $\omega \in \mathbf{U}$ and $k \in \mathbf{N}$. 
Proof of Theorem 1.5. By Theorem 1.4, there hold

$$
\frac{2 \pi}{k} i_{k \tau}=\frac{2 \pi}{k} \sum_{\omega^{k}=1} i_{\tau}(\omega), \quad \frac{2 \pi}{k} \nu_{k \tau}=\frac{2 \pi}{k} \sum_{\omega^{k}=1} \nu_{\tau}(\omega) .
$$

By Lemma 4.1, the function $i_{\tau}(\omega)$ is locally constant and $\nu_{\tau}(\omega)$ is locally zero on $\mathbf{U}$ except at finitely many points. Therefore the right hand sides of (5.6) are Riemannian sums, and converge to the corresponding integrals as $k \rightarrow \infty$.

As a direct consequence of Theorem 1.5, we obtain

$$
\hat{i}_{k \tau}=k \hat{i}_{\tau}, \quad \forall k \in \mathbf{N} .
$$

Next we give:

Proof of Theorem 1.6. Based on the results of splitting numbers in Section 4, the proof is completely parallel to that of Theorem 1.4 of $[\mathbf{R d}]$, and therefore is omitted.

Remark 5.2. Recently based on our above results, the following increasing estimate for any $\gamma \in \mathcal{P}_{\tau}(2 n)$ is proved by $\mathrm{C}$. Liu and the author in $[\mathbf{L L}]$,

$$
k \hat{i}_{\tau}(\gamma)-n \leq i_{k \tau}\left(\gamma^{k}\right) \leq k \hat{i}_{\tau}(\gamma)+n-\nu_{k \tau}\left(\gamma^{k}\right), \quad \forall k \in \mathbf{N} .
$$

This estimate is optimal on both sides of (5.8) as shown in [LL].

\section{Relations with other index functions.}

In this section we study the relationship between our $\omega$-index theory and the index functions of R. Bott defined in [Bo] and I. Ekeland defined in [Ek].

Firstly we consider the real periodic Sturm system:

$$
-\frac{d}{d t}\left(P \frac{d}{d t} x+Q x\right)+Q^{T} \frac{d}{d t} x+R x=0,
$$

where $P, Q$, and $R \in C^{1}\left(S_{\tau}, \mathcal{L}\left(\mathbf{R}^{n}\right)\right), P(t)$ and $R(t)$ are symmetric, and $P(t)$ is positive definite for all $t$. R. Bott defined his index function $\Lambda(\omega)$ and $N(\omega)$ in Section 1 of [Bo] for such systems and $\omega \in \mathbf{U}$. Define

$$
B(t) \equiv B_{x}(t)=\left(\begin{array}{cc}
P^{-1}(t) & -P^{-1}(t) Q(t) \\
-Q(t)^{T} P^{-1}(t) & Q(t)^{T} P^{-1}(t) Q(t)-R(t)
\end{array}\right) .
$$

Then our $\omega$-index theory is defined for the linear Hamiltonian system (1.2) with this $B(t)$, which we denote by $\left(i_{\tau}(\omega), \nu_{\tau}(\omega)\right)$ for $\omega \in \mathbf{U}$.

Theorem 6.1. Under the above assumptions, there hold

$$
i_{\tau}(\omega)=\Lambda(\omega), \quad \nu_{\tau}(\omega)=N(\omega), \quad \forall \omega \in \mathbf{U} .
$$

Proof. Note that the second equality holds by definition of the two sides. To prove the first equality, denote by $B S_{M}^{ \pm}(\omega)$ the splitting numbers defined on the page 180 of $[\mathbf{B o}]$. Then by a direct verification we obtain 
that $\left(B S_{M}^{+}(\omega), B S_{M}^{-}(\omega)\right)$ satisfies the four axioms in Corollary 4.14 for all $M \in \cup_{n \geq 1} \operatorname{Sp}(2 n)$. Therefore it coincides with our splitting numbers $\left(S_{M}^{+}(\omega)\right.$, $\left.S_{M}^{-}(\omega)\right)$. Thus we obtain

$$
\Lambda(\omega)-i_{\tau}(\omega)=\Lambda(1)-i_{\tau}(1) .
$$

By [Vi2] or $[\mathbf{L A}]$ we obtain that the right hand side of (6.4) is zero. Thus the first equality in (6.3) holds.

For $\tau>0$ denote by $\mathcal{E}_{\tau}(2 n)$ the set of $\gamma \in \hat{\mathcal{P}}_{\tau}(2 n)$ which are fundamental solutions of the systems (1.2) with negative definite $B(t)$ for all $t \in[0, \tau]$. In his celebrated work $[\mathbf{E k}]$, I. Ekeland systematically studied such Hamiltonian systems and defined his index theory via dual action principle. For any $\gamma \in E_{\tau}(2 n)$, denote the Ekeland index by $\left(j_{\tau, \omega}(\gamma), n_{\tau, \omega}(\gamma)\right)$ for all $\omega \in \mathbf{U}$ via Definition I.5.3 of $[\mathbf{E k}]$. Note that $i_{\tau}^{E}(\gamma)=j_{\tau, 1}(\gamma)$ and $\nu_{\tau}^{E}(\gamma)=n_{\tau, 1}(\gamma)$ is the Ekeland index defined by Definition I.4.3 of [Ek]. The following result give the relation between our index theory and Ekeland's.

Theorem 6.2. For any $\gamma \in \mathcal{E}_{\tau}(2 n)$, there hold

$$
\begin{aligned}
\nu_{\tau, \omega}(\gamma) & =n_{\tau, \omega}(\gamma), \quad \forall \omega \in \mathbf{U}, \\
i_{\tau, 1}(\gamma)+\nu_{\tau, 1}(\gamma) & =-j_{\tau, 1}(\gamma)-n, \\
i_{\tau, \omega}(\gamma)+\nu_{\tau, \omega}(\gamma) & =-j_{\tau, \omega}(\gamma), \quad \forall \omega \in \mathbf{U} \backslash\{1\} .
\end{aligned}
$$

We need:

Lemma 6.3. For $\tau>0$ and $\gamma \in \mathcal{E}_{\tau}(2 n)$, denote by $(p, p)$ the ultimate type of $1 \in \sigma(\gamma(\tau))$, by $m_{0}$ the total multiplicity of $1 \in \sigma(\gamma(\tau))$ and by $2 r_{0}^{-}$the number of Floquet multipliers which arrive on the unit circle at 1 along $\gamma$ as defined in the Definition I.3.6 of [Ek]. Then there holds

$$
\nu_{\tau}(\gamma)-p=\frac{m_{0}}{2}-r_{0}^{-} .
$$

Proof. Set $\tau=1$. Firstly note that the definition of $r_{0}^{-}$in $[\mathbf{E k}]$ can be localized, i.e. every short curve $\beta:[0,1] \rightarrow \operatorname{Sp}(2 n)$ satisfying $\beta(1)=$ $\gamma(1)$ and $-J \dot{\beta}(t) \beta(t)^{-1}$ being negative definite for all $t \in[0,1]$ possesses the same $r_{0}^{-}$, whose proof is left to the readers. By Theorem 4.11, $p=$ $S_{\gamma(1)}^{+}(1)$ satisfies Corollary 4.14. Thus together with Theorems 2.11, 2.13, and Theorem 7.8 for $M=\gamma(1)$, the proof of (6.8) is reduced to the case of $\gamma(1)=N_{1}(1, B)$ with $B= \pm 1$ or 0 . Then (6.8) of these three cases follows by direct computation. Note that the perturbation path $D(1+t \epsilon)$ in the cases 1 and 2 of the proof of Theorem 7.8 should be replaced by $D(1+t \epsilon) R(t \theta)$ with a small $-\theta>0$ and a much smaller $\epsilon>0$.

Proof of Theorem 6.2. Without loss of generality, we suppose $\tau=1$. Note that (6.5) follows from the Flouqet theory. 
Next we consider the proof of (6.6) when $\nu_{1}(\gamma)=0$. Similar to our discussions in Section 2, by a sufficiently small rotational perturbation, $\gamma$ is homotopic to a nearby $\diamond$-product path $\beta$ of paths $\beta_{j}$ in $\mathcal{E}_{1}(2) \cup \mathcal{E}_{1}(4)$ for $1 \leq$ $j \leq k$ with some $k \in[1, n]$ and each $\beta_{j}(1)$ possessing only simple eigenvalues. If $\beta_{j} \in \mathcal{E}_{1}(4)$, by extending it within $\mathcal{E}_{1}(4)$ to reach a matrix possessing a double eigenvalue pair on $\mathbf{R} \backslash\{0, \pm 1\}$, then by a small perturbation, similar to our discussions in Sections 2 and 4 we can use a $\diamond$-product of two paths in $\mathcal{E}_{1}(2)$ to replace this $\beta_{j}$. Thus without loss of generality, we can assume all $\beta_{j} \in \mathcal{E}_{1}(2)$. Note that Ekeland index for nondegenerate paths is constant under small perturbations and is symplectic additive. Therefore the proof of (6.6) for $\gamma$ is reduced to that for each $\beta_{j}$. Here we leave the details of the above discussion to the readers. By direct computation we obtain

$$
i_{\tau}\left(\beta_{j}\right)=-1-\sum_{0<t<1} \nu_{t}\left(\left.\beta_{j}\right|_{[0, t]}\right), \quad \text { for } \beta_{j} \in \mathcal{E}_{1}(2) .
$$

Thus (6.6) follows from Theorem I.4.6 of [Ek] and (6.9).

When $\nu_{1}(\gamma)>0$, let $\gamma_{s}$ for $s \in[-1,1]$ be the perturbation paths of $\gamma$ defined by (2.6). Since $\gamma \in \mathcal{E}_{1}(2 n)$, we can choose $\theta_{0}>0$ in (2.6) so small that $\gamma_{s} \in \mathcal{E}_{1}(2 n)$, and there holds

$$
i_{1}^{E}\left(\gamma_{-1}\right)-\nu_{1}^{E}(\gamma) \leq i_{1}^{E}(\gamma) \leq i_{1}^{E}\left(\gamma_{1}\right) .
$$

Thus by Theorem 2.5 and (6.6) for $\gamma_{s}$ with $s \neq 0$, we obtain

$$
i_{1}^{E}\left(\gamma_{1}\right)=i_{1}^{E}\left(\gamma_{-1}\right)-\nu_{1}^{E}(\gamma), \quad i_{1}^{E}(\gamma)=i_{1}^{E}\left(\gamma_{1}\right)
$$

Then together with (6.6) for $\gamma_{1}$ and Theorem 2.5, we obtain (6.6) for $\gamma$.

To prove $(6.7)$, by Theorem 4.11 , we obtain $\lim _{\epsilon \rightarrow 0^{+}} i_{1, \exp (\epsilon \sqrt{-1})}(\gamma)=$ $i_{1,1}(\gamma)+p$, where $(p, p)$ is the ultimate type of $1 \in \sigma(\gamma(1))$. By Proposition I.3.5 of $[\mathbf{E k}]$, there holds $\lim _{\epsilon \rightarrow 0^{+}} j_{1, \exp (\epsilon \sqrt{-1})}(\gamma)=j_{1,1}(\gamma)+n+\frac{m_{0}}{2}-r_{0}^{-}$. Thus together with Proposition I.5.11 of $[\mathbf{E k}]$, we obtain

$$
\begin{aligned}
\lim _{\epsilon \rightarrow 0^{+}} i_{1, \exp (\epsilon \sqrt{-1})}(\gamma) & =-j_{1,1}(\gamma)-n_{1,1}(\gamma)-n+p \\
& =-\left[j_{1,1}(\gamma)+n+\frac{m_{0}}{2}-r_{0}^{-}\right] \\
& =-\lim _{\epsilon \rightarrow 0^{+}} j_{1, \exp (\epsilon \sqrt{-1})}(\gamma) .
\end{aligned}
$$

Using (I.5.54) of [Ek] and our (4.2) we then obtain

$$
\lim _{\epsilon \rightarrow 0^{+}} i_{1, \exp (-\epsilon \sqrt{-1})}(\gamma)=-\lim _{\epsilon \rightarrow 0^{+}} j_{1, \exp (-\epsilon \sqrt{-1})}(\gamma) .
$$

Then (6.7) holds for all $\omega \in \mathbf{U} \backslash(\sigma(\gamma(1)) \cup\{1\})$ by Proposition I.5.9 of [Ek] and Theorem 4.11.

Suppose $\omega \in \sigma(\gamma(1)) \cap(\mathbf{U} \backslash\{1\})$. Choose the perturbation paths $\gamma_{s}$ with $s \in[-1,1]$ of $\gamma$ defined by (2.6) sufficiently close to $\gamma$ so that $\gamma_{s} \in \mathcal{E}_{1}(2 n)$ 
and there holds

$$
j_{1, \omega}\left(\gamma_{-1}\right)-\nu_{1, \omega}(\gamma) \leq j_{1, \omega}(\gamma) \leq j_{1, \omega}\left(\gamma_{1}\right)
$$

Similar to the proof of (6.6), using (6.7) for paths in $\mathcal{P}_{1, \omega}^{*}(2 n)$ and Theorem 2.5 we obtain

$$
j_{1, \omega}\left(\gamma_{1}\right)=-i_{1, \omega}\left(\gamma_{1}\right)=-i_{1, \omega}\left(\gamma_{-1}\right)-\nu_{1, \omega}(\gamma)=j_{1, \omega}\left(\gamma_{-1}\right)-\nu_{1, \omega}(\gamma) .
$$

Then we obtain

$$
j_{1, \omega}(\gamma)=j_{1, \omega}\left(\gamma_{1}\right)
$$

Then together with (6.7) for $\gamma_{1}$ and Theorem 2.5 we obtain

$$
\begin{aligned}
i_{1, \omega}(\gamma) & =i_{1, \omega}\left(\gamma_{-1}\right)=i_{1, \omega}\left(\gamma_{1}\right)-\nu_{1, \omega}(\gamma) \\
& =-j_{1, \omega}\left(\gamma_{1}\right)-\nu_{1, \omega}(\gamma)=-j_{1, \omega}(\gamma)-\nu_{1, \omega}(\gamma) .
\end{aligned}
$$

This proves (6.7) for $\omega \in \sigma(\gamma(1)) \cap(\mathbf{U} \backslash\{1\})$.

Corollary 6.4. For $\tau>0$ and $\gamma \in \mathcal{E}_{\tau}(2 n)$, denote by $\left(p^{0}, q^{0}\right)$ and $(p, q)$ the Krein type and the ultimate type of $\omega \in \sigma(\gamma(\tau)) \cap(\mathbf{U} \backslash\{1\})$ respectively, and by $2 r_{0}^{-}$the number of Floquet multipliers which arrive on the unit circle at $\omega$ along $\gamma$ as defined in Definition I.3.6 of [Ek]. Then there hold

$$
\nu_{\tau, \omega}(\gamma)-p=q^{0}-r_{0}^{-}, \quad \nu_{\tau, \omega}(\gamma)-q=p^{0}-r_{0}^{-} .
$$

Proof. This corollary follows from Proposition I.5.11 of [Ek] and Theorem 6.2 .

As Lemma 6.3, (6.10) can also be proved directly.

Now from Theorems 1.4 and 6.1, we recover the Bott formula for the Morse index theory of closed geodesics. From Theorems 1.4 and 6.2 we recover the Bott-type formula Corollary I.5.4 of [Ek] for the Ekeland index theory of convex Hamiltonian systems. By Theorems 1.5 and 6.2 the corresponding mean indices satisfy $\hat{i}_{\tau}(x)=-\hat{i}_{\tau}^{E}(x)$.

Remark 6.5. Our choice of the standard symplectic matrix $J$ on $\mathbf{R}^{2 n}$ in Section 1 coincides with those in $[\mathbf{B o}],[\mathbf{R a}],[\mathbf{C Z}],[\mathbf{L Z}],[\mathbf{L o 1}]-[\mathbf{L o 9}],[\mathbf{D L}]$ and $[\mathbf{L D}]$. Note that there is a sign difference of this matrix between our choice and $[\mathbf{Y S}]$ and $[\mathbf{E k}]$. Because of this reason, there is a sign difference between our Corollary 4.12 and Proposition I.5.9 of [Ek]. If we take the same $J$ as defined in Section 1 to be the standard symplectic matrix, then (6.6) should be replaced by $i_{\tau}(\gamma)=i_{\tau}^{E}(\gamma)+n$ for all $\gamma \in \mathcal{E}_{\tau}(2 n)$, which was proved by Theorem II.C.1 of $[\mathbf{B r}]$ when $\nu_{\tau}(\gamma)=0$ and by Lemma 1.3 of [Lo7] when $\nu_{\tau}(\gamma)>0$. In $[\mathbf{B r}]$, the Conley-Zehnder index theory $i_{T}$ defined in $[\mathbf{C Z}]$ is extended with $J$ being replaced by $-J$ to the degenerate linear Hamiltonian systems by Definition II.D.1 of [Br], and two extensions $i_{T}^{ \pm}=m_{1 / 2, T}^{ \pm}$of $i_{T}$ are obtained. In Theorem III.C.1 of $[\mathbf{B r}]$, the indices $i_{T}^{ \pm}$are extended to functions $j^{ \pm}: \mathbf{U} \rightarrow \mathbf{Z}$, and the corresponding Bott-type formulae (III.C.5.1) and (III.C.5.2) of $[\mathbf{B r}]$ for $i_{T}^{ \pm}$in terms of $j^{ \pm}$are claimed without any proofs. 
These two formulae are incorrect, since they make no distinction between trivial and nontrivial normal forms. To see this for (III.C.5.1) of [Br], let $T=1$ and $H(t)=\operatorname{diag}(\pi / 2, \pi / 2)$ for all $t \in[0,1]$ in the notations of $[\mathbf{B r}]$. Then there holds $i_{4}^{+}=3 \neq 1=\sum_{\omega^{4}=1} j^{+}(\omega)$. For (III.C.5.2) of [Br], one can consider $H(t)=\left(h_{i, j}\right)$ with $h_{2,2}=1$ or -1 , and all other $h_{i, j}=0$ with $1 \leq i, j \leq 2$.

\section{Normal forms and homotopy components.}

In this section we briefly recall results obtained in [Lo6] on topological structures of $\omega$-subsets, in [LD] on normal forms for eigenvalues in $\mathbf{U}$, and study the homotopy components for symplectic matrices.

Given any two symplectic matrices of square block form:

$$
M_{1}=\left(\begin{array}{ll}
A_{1} & B_{1} \\
C_{1} & D_{1}
\end{array}\right)_{2 i \times 2 i}, \quad M_{2}=\left(\begin{array}{ll}
A_{2} & B_{2} \\
C_{2} & D_{2}
\end{array}\right)_{2 j \times 2 j},
$$

we define an operation $\diamond$-product of $M_{1}$ and $M_{2}$ to be the $2(i+j) \times 2(i+j)$ matrix $M_{1} \diamond M_{2}$ given by

$$
M_{1} \diamond M_{2}=\left(\begin{array}{cccc}
A_{1} & 0 & B_{1} & 0 \\
0 & A_{2} & 0 & B_{2} \\
C_{1} & 0 & D_{1} & 0 \\
0 & C_{2} & 0 & D_{2}
\end{array}\right) .
$$

Denote by $M^{\diamond k}$ the $k$-fold $\diamond$-product $M \diamond \cdots \diamond M$. Note that the $\diamond$-multiplication is associative, and the $\diamond$-product of any two symplectic matrices is symplectic. For $\theta \in \mathbf{R}$ and $a \in \mathbf{R} \backslash\{0\}$ denote by $D(a)=\operatorname{diag}(a, 1 / a)$, $R(\theta)=\left(\begin{array}{cc}\cos \theta & -\sin \theta \\ \sin \theta & \cos \theta\end{array}\right)$, and $R_{k}(\theta)=I_{2 k-2} \diamond R(\theta) \diamond I_{2 n-2 k}$.

For any $\omega \in \mathbf{U}$ and $M \in \operatorname{Sp}(2 n)$ define

$$
D_{\omega}(M)=(-1)^{n-1} \omega^{-n} \operatorname{det}(M-\omega I) .
$$

Then $D_{\omega}=D_{\bar{\omega}}$ for all $\omega \in \mathbf{U}$ and $D \in C^{\infty}(\mathbf{U} \times \operatorname{Sp}(2 n), \mathbf{R})$. For $\omega \in$ $\mathbf{U}$, define $\operatorname{Sp}(2 n)_{\omega}^{0}=\left\{M \in \operatorname{Sp}(2 n) \mid D_{\omega}(M)=0\right\}, \operatorname{Sp}(2 n)_{\omega}^{ \pm}=\{M \in$ $\left.\operatorname{Sp}(2 n) \mid \pm D_{\omega}(M)<0\right\}$, and $\operatorname{Sp}(2 n)_{\omega}^{*}=\operatorname{Sp}(2 n)_{\omega}^{+} \cup \operatorname{Sp}(2 n)_{\omega}^{-}$. There hold $\operatorname{Sp}(2 n)_{\omega}^{0}=\operatorname{Sp}(2 n) \frac{0}{\omega}$ and $\operatorname{Sp}(2 n)_{\omega}^{*}=\operatorname{Sp}(2 n)_{\omega}^{*}$. Define $M_{n}^{+}=D(2)^{\diamond n}, M_{n}^{-}=$ $D(-2) \diamond D(2)^{\diamond(n-1)}$, and

$\mathcal{M}_{\omega}^{k}(2 n)=\left\{M \in \operatorname{Sp}(2 n)_{\omega}^{0} \mid \operatorname{dim}_{\mathbf{C}} \operatorname{ker}_{\mathbf{C}}(M-\omega I)=k\right\}, \quad$ for $k=1, \ldots, 2 n$.

An intuitive picture of these sets can be found in [Lo2] and Figure 4.1 by the $\mathbf{R}^{3}$-cylindrical representation of $\operatorname{Sp}(2)$ introduced in [Lo2].

Theorem 7.1 (cf. [Lo6]). For any $\omega \in \mathbf{U}$, the $\omega$-nonsingular set $\operatorname{Sp}(2 n)_{\omega}^{*}$ contains exactly two path-connected components $\operatorname{Sp}(2 n)_{\omega}^{+}$and $\operatorname{Sp}(2 n)_{\omega}^{-}$and 
there holds $M_{n}^{ \pm} \in \mathrm{Sp}(2 n)_{\omega}^{ \pm}$. $\mathrm{Sp}(2 n)_{\omega}^{*}$ is simply connected in $\mathrm{Sp}(2 n)$ for any $\omega \in \mathbf{U}$.

Theorem 7.2 (cf. [Lo6]). For any $M \in \mathcal{M}_{\omega}^{1}(2 n)$ with $\omega \in \mathbf{U} \backslash \mathbf{R}$, by Theorem 7.1 there exists $P \in \operatorname{Sp}(2 n)$ such that

$$
P M P^{-1}=N_{k}(\hat{\omega}, B) \diamond M_{1} \equiv N,
$$

with $\hat{\omega} \equiv e^{\theta \sqrt{-1}}=\omega$ or $\bar{\omega}$, and $M_{1} \in \operatorname{Sp}(2 h)_{\omega}^{*}$ with $h+k=n$. Then there exist $M_{0} \in \operatorname{Sp}(2 n-2)_{\omega}^{*}$ and a continuous path $\sigma:[0,1] \rightarrow \mathcal{M}_{\omega}^{1}(2 n)$ such that

$$
\sigma(0)=M, \quad \sigma(1)=R(\theta) \diamond M_{0} .
$$

Moreover for any sufficiently small $\alpha>0$, there exist continuous paths $\sigma^{ \pm}$: $[0,1] \rightarrow \operatorname{Sp}(2 n)_{\omega}^{*}$ such that

$$
\begin{aligned}
\sigma^{-}(0) & =M P^{-1} R_{1}(-\alpha) P, & \sigma^{-}(1) & =[R(\theta) R(-\alpha)] \diamond M_{0}, \\
\sigma^{+}(0) & =M P^{-1} R_{1}(\alpha) P, & \sigma^{+}(1) & =[R(\theta) R(\alpha)] \diamond M_{0},
\end{aligned}
$$

and the distance between $\sigma^{ \pm}$and $\sigma$ is not greater than twice of the distance between $N$ and $N R_{1}(\alpha)$.

Next we consider normal forms of symplectic matrices for eigenvalues on $\mathbf{U}$ studied in $[\mathbf{L D}]$.

Case 1. The normal form $N_{k}( \pm 1, B) \in \mathcal{M}_{ \pm 1}^{1}(2 k) \cup \mathcal{M}_{ \pm 1}^{2}(2 k)$.

For $\lambda= \pm 1$, we define

$$
N_{1}(\lambda, B)=\left(\begin{array}{cc}
\lambda & B \\
0 & \lambda
\end{array}\right), \quad \text { for } B= \pm 1, \text { or } 0 .
$$

For $k \geq 2$, the matrix $N_{k}(\lambda, b)$ is of the following form with $k \times k$ real matrices $A(\lambda), B(\lambda, b), C(\lambda)$, and $B=\left(b_{1}, \ldots, b_{k}\right) \in \mathbf{R}^{k}$ :

$$
N_{k}(\lambda, B)=\left(\begin{array}{cc}
A_{k}(\lambda) & B_{k}(\lambda, B) \\
0 & C_{k}(\lambda)
\end{array}\right)
$$

where $A_{k}(\lambda)=\left(A_{i, j}\right)$ is a Jordan block of $\lambda$, i.e. $A_{i, i}=\lambda, A_{i, i+1}=1$, and all other $A_{i, j}=0 ; B_{k}(\lambda, B)=\left(B_{i, j}\right)$ is a lower triangle matrix satisfying $B_{i, j}=0$ if $i<j$ and $B_{i, j}=(-\lambda)^{j-1} b_{i}$ if $i \geq j ; C_{k}(\lambda)=\left(C_{i, j}\right)$ is a lower triangle matrix satisfying $C_{i, j}=0$ if $i<j$ and $C_{i, j}=-(-\lambda)^{i-j+1}$ if $i \geq j$.

Case 2. The normal form $N_{2 k}(\omega, B) \in \mathcal{M}_{\omega}^{1}(4 k) \cup \mathcal{M}_{\omega}^{2}(4 k)$ for the eigenvalue pair $\{\omega, \bar{\omega}\}$ with $\omega=e^{\theta \sqrt{-1}} \in \mathbf{U} \backslash \mathbf{R}$.

The matrix $N_{2 k}(\omega, B)$ is of the following form with $2 k \times 2 k$ real matrices $A_{2 k}(\omega), B$, and $C_{2 k}(\omega)$ :

$$
N_{2 k}(\omega, B)=\left(\begin{array}{cc}
A_{2 k}(\omega) & B \\
0 & C_{2 k}(\omega)
\end{array}\right)
$$


where $A_{2 k}(\omega)=\left(A_{i, j}\right)$ and $C_{2 k}(\omega)=\left(C_{i, j}\right)$ are $2 \times 2$ blockwise blockwise lower triangular matrices and satisfy $A_{i, i}=R(\theta), A_{i, i+1}=I_{2}$, all other $A_{i, j}=0 ; C_{i, j}=0$ if $i<j$, and $C_{i, j}=-(-1)^{i-j} R((i-j+1) \theta)$ if $i \geq j$.

Case 3. The normal form $N_{k}(\omega, B) \in \operatorname{Sp}(2 k)$ for the eigenvalue pair $\{\omega, \bar{\omega}\}$ with $\omega=e^{\theta \sqrt{-1}} \in \mathbf{U} \backslash \mathbf{R}$ and $k \in 2 \mathbf{N}-1$.

When $k=1, N_{1}(\omega, 0)=R(\theta)$ and $N_{1}(\bar{\omega}, 0)=R(2 \pi-\theta) \in \mathcal{M}_{\omega}^{1}(2)$.

When $k=2 m+1 \geq 2$, the following matrix $N_{k}(\omega, B) \in \mathcal{M}_{\omega}^{1}(2 k)$ with $2 m \times 2 m$ matrices $A_{2 m}(\theta), B, C_{2 m}(\theta)$, defined in (7.5), and $2 m \times 1$ matrices $D, E, F, G$ :

$$
N_{k}(\omega, B)=\left(\begin{array}{cccc}
A_{2 m}(\omega) & D & B & E \\
0 & \cos \hat{\theta} & F^{T} & -\sin \hat{\theta} \\
0 & 0 & C_{2 m}(\omega) & 0 \\
0 & \sin \hat{\theta} & G^{T} & \cos \hat{\theta}
\end{array}\right),
$$

and

$$
\left\{\begin{array}{l}
\hat{\theta}=\theta, \quad D=(0, \ldots, 0,1,0)^{T}, E=(0, \ldots, 0,0,1)^{T}, \quad \text { or } \\
\hat{\theta}=-\theta, \quad D=(0, \ldots, 0,0,1)^{T}, E=(0, \ldots, 0,1,0)^{T} .
\end{array}\right.
$$

The case of $k=2 m \geq 2$ : The matrix $N_{k}(\omega, B) \in \mathcal{M}_{\omega}^{2}(2 k)$ is given by (7.5), or by

$$
N_{k}(\omega, B)=\left(\begin{array}{cc}
N_{m}\left(\omega, B_{1}\right) & B_{0} \\
0 & N_{m}\left(\omega, B_{2}\right)
\end{array}\right),
$$

with $N_{m}\left(\omega, B_{i}\right)$ for $i=1$ and 2 given by (7.6).

Definition 7.3. The normal form $N_{n}(\omega, B) \in \operatorname{Sp}(2 n)$ belonging to the eigenvalue $\omega \in \mathbf{U}$ possesses the type number $t\left(N_{n}(\omega, B)\right)=1$ if $N_{n}(\omega, B)\left[R(\alpha) \diamond I_{2 n-2}\right] \in \operatorname{Sp}(2 n)_{\omega}^{*}$ for all sufficiently small $|\alpha|>0$, and $t\left(N_{n}(\omega, B)\right)=2$ if $N_{n}(\omega, B)\left[R(\alpha) \diamond I_{2 n-2}\right] \in \mathrm{Sp}(2 n)_{\omega}^{0}$ for all $\alpha \in \mathbf{R}$.

Remark 7.4. Note that when $t\left(N_{n}(\omega, B)\right)=2$, there must hold $N_{n}(\omega, B) \in$ $\mathcal{M}_{\omega}^{2}(2 n), \quad N_{n}(\omega, B)\left[R(\alpha) \diamond I_{2 n-2}\right] \in \mathcal{M}_{\omega}^{1}(2 n)^{0}, \quad N_{n}(\omega, B)\left[I_{2 n-2} \diamond R(\alpha)\right] \in$ $\mathcal{M}_{\omega}^{1}(2 n)^{0}$, and

$$
N_{n}(\omega, B)\left[R(\alpha) \diamond I_{2 n-4} \diamond R(\alpha)\right] \in \operatorname{Sp}(2 n)_{\omega}^{*}
$$

for all sufficiently small $|\alpha|>0$. Note also that $t\left(N_{n}(\omega, B)\right)=2$ if and only if $\omega= \pm 1$ and $n \geq 2$, or $\omega \in \mathbf{U} \backslash \mathbf{R}$ and $n \geq 3$.

Theorem 7.5 (cf. $[\mathbf{L D}]$ ). For any $M \in \operatorname{Sp}(2 n)$ and $\omega=e^{\theta \sqrt{-1}} \in \mathbf{U}$, there exist $P \in \operatorname{Sp}(2 n)$ and two nonnegative integers $p$ and $q$ satisfying $1 \leq p+q \leq n$, first type normal forms $H_{i} \in \mathrm{Sp}\left(2 h_{i}\right)$ and second type normal forms $K_{j} \in \operatorname{Sp}\left(2 k_{j}\right)$ belonging to the eigenvalue $\omega$ for $1 \leq i \leq p$ and $1 \leq$ 
$j \leq q$ defined above, and $M_{0} \in \operatorname{Sp}(2 n-2 m)_{\omega}^{*}$ with $m=\sum_{i=1}^{p} h_{i}+\sum_{i=1}^{q} k_{i}$, such that there holds

$$
P M P^{-1}=H_{1} \diamond \cdots \diamond H_{p} \diamond K_{1} \diamond \cdots \diamond K_{q} \diamond M_{0} .
$$

In this case setting $k_{0}=h_{0}=0$, we define

$$
\begin{cases}m_{i}=\sum_{s=0}^{i-1} h_{s}+1, & \text { for } 1 \leq i \leq p, \\ m_{p+2 j-1}=\sum_{s=0}^{p} h_{s}+\sum_{s=0}^{j-1} k_{s}+1, & \text { for } 1 \leq j \leq q, \\ m_{p+2 j}=\sum_{s=0}^{p} h_{s}+\sum_{s=0}^{j-1} k_{s}+k_{j}, & \text { for } 1 \leq j \leq q .\end{cases}
$$

Theorem 7.6 (cf. $[\mathbf{L D}]$ ). For any $M \in \operatorname{Sp}(2 n)$, there exist $P \in \operatorname{Sp}(2 n)$, integer $p \in[0, n]$, and normal forms $M_{j} \in \operatorname{Sp}\left(2 k_{j}\right)$ for $1 \leq j \leq p$ belonging to eigenvalues on $\mathbf{U}, M_{0} \in \operatorname{Sp}\left(2 n-\sum_{j=1}^{p} k_{j}\right)$ satisfying $\sigma\left(M_{0}\right) \cap \mathbf{U}=\emptyset$, such that there holds

$$
P M P^{-1}=M_{1} \diamond \cdots \diamond M_{p} \diamond M_{0} .
$$

Next using normal forms, we study the homotopy components. For any $M \in \mathrm{Sp}(2 n)$, to find much simpler elements in $\Omega^{0}(M)$ or $\Omega_{\omega}^{0}(M)$ than (7.10).

Case 1. $M=N_{n}(1, B) \in \mathcal{M}_{1}^{1}(2 n)$ with $n \geq 2$.

For $\epsilon>0$ small enough, we define

$$
f(t)=M\left[I_{2} \diamond D(1+t \epsilon)^{\diamond(n-1)}\right], \quad \forall t \in[0,1] .
$$

Applying Theorem 7.6 to $f(1)$, we obtain $P \in \operatorname{Sp}(2 n)$ such that $P f(1) P^{-1}=$ $N_{1}(\lambda, c) \diamond M_{0}$ with $\sigma\left(M_{0}\right) \cap \mathbf{U}=\emptyset$. Together with Theorem 7.1, $M$ can be connected to $N_{1}(1, c) \diamond M_{0}$ within $\Omega^{0}(M)$.

Case 2. $\quad M=N_{n}(1, B) \in \mathcal{M}_{\lambda}^{2}(2 n)$ with $n \geq 2$.

We define $f(t)=N_{n}(1,(1-t) B)$ for $t \in[0,1]$ and

$$
g(t)=f(1)\left[I_{2} \diamond D(1+t)^{\diamond(n-2)} \diamond I_{2}\right], \quad \forall t \in[0,1] .
$$

Applying Theorem 7.6 to $g(1)$, we obtain a path $h$ in $\Omega_{1}(g(1))$ which connects $g(1)$ to $N_{2}(1, c) \diamond M_{0}$ with $\sigma\left(M_{0}\right) \cap \mathbf{U}=\emptyset$. Define $4 \times 4$ matrix paths $\phi(t)=$ $N_{2}(1,(1-t) c)$ and $\psi(t)=\left(\psi_{i, j}(t)\right)$ with $\psi_{1,1}(t)=\psi_{3,3}(t)=1, \psi_{2,2}(t)=$ $\psi_{4,4}(t)^{-1}=1+t, \psi_{1,2}(t)=1-t^{2}, \psi_{4,3}(t)=t-1$, and all other $\psi_{i, j}(t)=0$ for all $t \in[0,1]$. Then the path $\left[(\psi * \phi) \diamond M_{0}\right] * h * g * f$ connects $M$ to $I_{2} \diamond D(2) \diamond M_{0}$ in $\Omega^{0}(M)$.

Case 3. $\quad M=N_{n}(\omega, B) \in \mathcal{M}_{\omega}^{1}(2 n)$ is a normal form of the eigenvalue $\omega \in \mathbf{U} \backslash \mathbf{R}$.

For $\epsilon>0$ small enough, we define

$$
f(t)=N_{n}(\omega, B)\left[D(1+t \epsilon)^{\diamond(n-k)} \diamond I_{2 k}\right], \quad \forall t \in[0,1],
$$

where $k=2$ if $M$ is given by (7.5) or (7.7), and $k=1$ if $M$ is given by (7.6). Then $f(t) \in \Omega^{0}(M)$ for all $t \in[0,1]$. Thus together with Theorem 
7.6, $M$ can be connected to $N_{k}(\hat{\omega}, c) \diamond M_{0}$ within $\Omega^{0}(M)$ with $\hat{\omega}=\omega$ or $\bar{\omega}$, $\sigma\left(M_{0}\right) \cap \mathbf{U}=\emptyset$, and some $c$.

Case 4. $\quad M=N_{n}(\omega, B) \in \mathcal{M}_{\omega}^{2}(2 n)$ is a normal form of the eigenvalue $\omega \in \mathbf{U} \backslash \mathbf{R}$ given by $(7.5)$.

We define $f(t)=N_{n}(\omega,(1-t) B)$ and

$$
g(t)=f(1)\left[D(1+t)^{\diamond(n-2)} \diamond I_{4}\right], \quad \forall t \in[0,1] .
$$

Then there exists a path $h$ in $\mathcal{M}_{\omega}^{2}(2 n)$ which connects $g(1)$ to $N_{2}(\hat{\omega}, 0) \diamond M_{0}$ with $\hat{\theta}=\theta$ or $2 \pi-\theta$ and $M_{0} \in \operatorname{Sp}(2 n-4)$ satisfies $\sigma\left(M_{0}\right) \cap \mathbf{U}=\emptyset$. We define

$$
k(t)=\left(\begin{array}{cccc}
c & -s(1-t) & -s t & s \sqrt{2 t-2 t^{2}} \\
s(1-t) & c & s \sqrt{2 t-2 t^{2}} & s t \\
s t & -s \sqrt{2 t-2 t^{2}} & c & -s(1-t) \\
-s \sqrt{2 t-2 t^{2}} & -s t & s(1-t) & c
\end{array}\right), \forall t \in[0,1],
$$

where $c=\cos \hat{\theta}$ and $s=\sin \hat{\theta}$. By direct computation we obtain $k(t) \in$ $\mathcal{M}_{\omega}^{2}(4)$ for all $t \in[0,1]$. Note that the path $k$ connects $N_{2}(\hat{\omega}, 0)$ to $R(\hat{\theta}) \diamond R(-\hat{\theta})$ within $\mathcal{M}_{\omega}^{2}(4)$. Thus there is a path in $\Omega^{0}(M)$ which connects $M$ to $R(\hat{\theta}) \diamond R(-\hat{\theta}) \diamond M_{0}$.

Note that $N_{n}(\omega, B)$ for $\omega \in \mathbf{U}$ in other cases can also be connected to $\diamond$-product of basic normal forms defined below and matrices possessing no eigenvalues on $\mathbf{U}$. Since the proofs are similar, they are omitted.

Definition 7.7. A normal form matrix $M=N_{n}(\omega, B) \in \operatorname{Sp}(2 n)$ defined above is a basic normal form if $n=1$ and $\omega \in \mathbf{U}$, or $M=N_{2}(\omega, B) \in$ $\mathcal{M}_{\omega}^{1}(4)$ and $\omega \in \mathbf{U} \backslash \mathbf{R}$.

Together with Theorem 7.6 and our above discussion, we obtain the following result:

Theorem 7.8. For any $M \in \operatorname{Sp}(2 n)$, there is a path $f \in C^{\infty}\left([0,1], \Omega^{0}(M)\right)$ such that $f(0)=M$ and

$$
f(1)=M_{1} \diamond \cdots \diamond M_{p} \diamond M_{0},
$$

where the integer $p \in[0, n]$, each $M_{i}$ is a basic normal form of eigenvalues on $\mathbf{U}$ for $1 \leq i \leq p$, and the symplectic matrix $M_{0}$ satisfies $\sigma\left(M_{0}\right) \cap \mathbf{U}=\emptyset$.

Lemma 7.9 (cf. [Lo6]). For $A$ and $B \in \mathbf{R}$ suppose the polynomial equation,

$$
\lambda^{4}-4 A \lambda^{3}+B \lambda^{2}-4 A \lambda+1=0,
$$

possesses no real roots. Then (7.13) possesses two pairs of conjugate simple roots on $\mathbf{U}$ if and only if $4 A^{2}+2>B$. (7.13) possesses one pair of conjugate double roots on $\mathbf{U}$ if and only if $4 A^{2}+2=B$. (7.13) possesses four simple roots away from $\mathbf{U} \cup \mathbf{R}$ if and only if $4 A^{2}+2<B$. 
Proof. By direct computation.

Lemma 7.10 (cf. [Lo6]). For $\omega=e^{\theta \sqrt{-1}}$ with $\theta \in(0,2 \pi) \backslash\{\pi\}$ and $B=$ $\left(\begin{array}{ll}b_{1} & b_{2} \\ b_{3} & b_{4}\end{array}\right) \in \mathcal{L}\left(\mathbf{R}^{2}\right)$, consider the matrix $M=N_{2}(\omega, B)$.

$1^{\circ} \quad M \in \operatorname{Sp}(4)$ if and only if $\left(b_{2}-b_{3}\right) \cos \theta+\left(b_{1}+b_{4}\right) \sin \theta=0$. In the following we always suppose $M \in \operatorname{Sp}(4)$.

$2^{\circ} \omega=\cos \theta+\sqrt{-1} \sin \theta$ and $\bar{\omega}$ are double eigenvalues of $M . M \in \mathcal{M}_{\omega}^{1}(4)$ if and only if $b_{2}-b_{3} \neq 0$, and $M \in \mathcal{M}_{\omega}^{2}(4)$ if and only if $b_{2}-b_{3}=0$.

$3^{\circ}$ For $M \in \mathcal{M}_{\omega}^{1}(4)$, there exists $\alpha_{0}>0$ small enough such that $M\left(R(\alpha) \diamond I_{2}\right) \in \operatorname{Sp}(4)_{\omega}^{*}$ if $0<|\alpha| \leq \alpha_{0}$.

$4^{\circ}$ Any $M \in \mathcal{M}_{\omega}^{1}(4)$ can be connected within $\mathcal{M}_{\omega}^{1}(4)$ to $N_{2}(\omega, D)$ such that for any $\epsilon>0$ there exists a perturbation path $\gamma:[0,1] \rightarrow \mathcal{M}_{\omega}^{1}(4)$ such that $\gamma(0)=N_{2}(\omega, D), \sigma(\gamma(t))$ possesses two pairs of simple eigenvalues $\{\omega, \bar{\omega}, \lambda(t), \overline{\lambda(t)}\}$ and $\lambda \in C([0,1], \mathbf{U})$ satisfies $\lambda(0)=\omega, \lambda(t) \neq \omega$ and $|\lambda(t)-\omega| \leq \epsilon$ for $0<t \leq 1$.

$5^{\circ}$ For $M \in \mathcal{M}_{\omega}^{2}(4)$, there holds $D_{\omega}\left(M\left[R(\alpha) \diamond I_{2}\right]\right)=0$ if and only if $\alpha=0 \bmod 2 \pi$.

Corollary 7.11. For $M \in \mathrm{Sp}(2 n)$ and $\omega \in \mathbf{U}$, there exist a path $f$ : $[0,1] \rightarrow \Omega_{\omega}^{0}(M)$ such that $f(0)=M$ and

$$
f(1)=M_{1} \diamond \cdots \diamond M_{n} .
$$

Here for $1 \leq i \leq n$, each $M_{i}$ is of the form $N_{1}(\omega, B), N_{1}(\bar{\omega}, B), D(2)$, or $D(-2)$ for $1 \leq i \leq n$.

Proof. This corollary follows from Theorems 7.1, 7.8, and Lemma 7.10.

Aknowledgements. The first manuscript [Lo9] of this paper appeared in Fall 1995, which has contained the main idea of the current version of this paper. Since then, specially during September to December 1996, I discussed with Professor Nancy Hingston on this manuscript by email and mail many times, and tried to explain to her my idea and certain details of this paper. I would like to sincerely thank her for her careful reading and pointing out some mistakes in the manuscript of this paper.

\section{References}

[BTZ] W. Ballmann, G. Thobergsson and W. Ziller, Closed geodesics on positively curved manifolds, Ann. of Math., 116 (1982), 213-247.

[Be] V. Benci, A new apporoach to the Morse-Conley theory and some applications, Ann. di Mat. pura ed appl., CLVIII (1991), 231-305.

[Bo] R. Bott, On the iteration of closed geodesics and the Sturm intersection theory, Comm. Pure Appl. Math., 9 (1956), 171-206.

[Br] V. Brousseau, Espaces de Krein et index des systemes hamiltoniens, Ann. Inst. H. Poincaré Anal non linéaire, 7 (1990), 525-560. 
[Ch] K.C. Chang, Infinite Dimensional Morse Theory and Multiple Solution Problems, Birkhäuser, Boston, 1993.

[CZ] C. Conley and E. Zehnder, Morse-type index theory for flows and periodic solutions for Hamiltonian equations, Comm. Pure Appl. Math., 37 (1984), 207-253.

[CD] R. Cushman and J.J. Duistermaat, The behavior of the index of a periodic linear Hamiltonian system under iteration, Adv. in Math., 23 (1977), 1-21.

[DL] D. Dong and Y. Long, The iteration formula of the Maslov-type index theory with applications to nonlinear Hamiltonian systems, Nankai Inst. of Math., Nankai Univ., preprint, 1993; ICTP. Report. No. IC/94/318, 1994, Trans. Amer. Math. Soc., 349 (1997), 2619-2661.

[Ed] H.M. Edwards, A generalized Sturm theorem, Ann. of Math., 80 (1964), 22-57.

[Ek] I. Ekeland, Convexity Methods in Hamiltonian Mechanics, Springer, Berlin, 1990.

[Kl] W. Klingenberg, Lectures on closed geodesics, Springer, Berlin, 1978.

[LL] C. Liu and Y. Long, An optimal increasing estimate for Maslov-type indices of iterations, Nankai Inst. of Math., Nankai Univ., preprint, 1997; Chinese Bull. Sci., 42 (1997) 2275-2278.

[Lo1] Y. Long, Maslov-type index, degenerate critical points, and asymptotically linear Hamiltonian systems, Science in China (Scientia Sinica), Series A, 33 (1990), 14091419.

[Lo2] - The structure of the singular symplectic matrix set, Science in China (Scientia Sinica), Series A, 5 (1991), 457-465 (Chinese edition); 34 (1991), 897-907 (English edition).

[Lo3] _ A Maslov-type index theory and asymptotically linear Hamiltonian systems, in 'Dyna. Syst. and Rel. Topics', K. Shiraiwa ed., World Sci., (1991), 333-341.

[Lo4] , The Index Theory of Hamiltonian Systems with Applications, (in Chinese), Science Press, Beijing, 1993.

[Lo5] _ Periodic points of Hamiltonian diffeomorphisms on tori and a conjecture of C. Conley, ETH-Zürich Preprint, Dec. 1994.

[Lo6] - The topological structures of $\omega$-subsets of symplectic groups, Nankai Inst. of Math., Nankai Univ., preprint, 1997; Acta Math. Sinica, to appear.

[Lo7] - Hyperbolic closed characteristics on compact convex smooth hypersurfaces in $\mathbf{R}^{2 n}$, Nankai Inst. of Math., Nankai Univ., preprint, 1996; J. Diff. Equa., to appear.

[Lo8] _ A Maslov-type index theory for symplectic paths, Nankai Inst. of Math., Nankai Univ., preprint, 1997, Top. Meth. Nonl. Anal., 10 (1997), 47-78.

[Lo9] - The Bott formula of the Maslov-type index theory, Research Report, Nankai Inst. of Math., Nankai Univ., (1995, 1996).

[LA] Y. Long and T. An, Indexing domains of instability for Hamiltonian systems, Nodea, to appear.

[LD] Y. Long and D. Dong, Normal forms of symplectic matrices, Nankai Inst. of Math., Nankai Univ., preprint, (1995, 1997); Acta Math. Sinica, to appear.

[LZ] Y. Long and E. Zehnder, Morse theory for forced oscillations of asymptotically linear Hamiltonian systems, in 'Stoc. Proc. Phys. and Geom., S. Albeverio et al. ed., World Sci., (1990), 528-563. 
[Ra] P. Rabinowitz, Periodic solutions of Hamiltonian systems, Comm. Pure Appl. Math., 31 (1978), 157-184.

[Rd] H.-B. Rademacher, On the average indices of closed geodesics, J. Diff. Geod., 29 (1989), 65-83.

[SZ] D. Salamon and E. Zehnder, Morse theory for periodic solutions of Hamiltonian systems and the Maslov index, Comm. Pure and Appl. Math., 45 (1992), 1303-1360.

[Vi1] C. Viterbo, Equivariant Morse theory for starshaped Hamiltonian systems, Trans. Amer. Math. Soc., 311 (1989), 621-655.

[Vi2] _ A new obstruction to embedding Lagrangian tori, Invent. Math., 100 (1990), 301-320.

[YS] V. Yakubovich and V. Starzhinskii, Linear Differential Equations with Periodic Coefficients, New York, John Wiley \& Sons, 1975.

Received August 25, 1997 and revised December 14, 1997. The author was partially supported by the NNSF and MCSEC of China and the Qiu Shi Sci. Tech. Foundation. The author is also an associate member of ICTP.

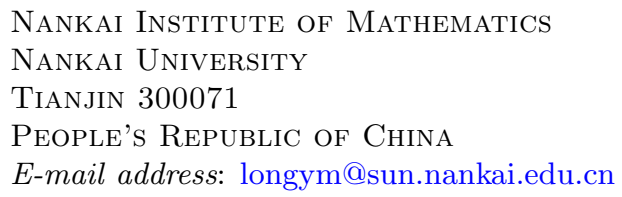

